\title{
Steady-State Solutions in Nonlocal Neuronal Networks
}

\author{
Linghai Zhang
}

Communicated by Charles Li, January 5, 2005

ABSTRACT. We focus on the mathematical analysis of existence and nonlinear
exponential stability (linear exponential instability, respectively) of steady-
state solutions of the scalar nonlocal equation
$\frac{\partial u}{\partial t}+u=(\alpha-\gamma u) \int_{\mathbb{R}} K(x-y) H(u(y, t)-\theta) d y+(\beta-\delta u) \int_{\mathbb{R}} K(x-y) H(u(y, t)-\Theta) d y$,

and the nonlinear system of integral-differential equations

$$
\begin{aligned}
\frac{\partial u}{\partial t}+u+w & =(\alpha-\gamma u) \int_{\mathbb{R}} K(x-y) H(u(y, t)-\theta) d y \\
& +(\beta-\delta u) \int_{\mathbb{R}} K(x-y) H(u(y, t)-\Theta) d y \\
\frac{\partial w}{\partial t} & =\varepsilon(u-\tau w) .
\end{aligned}
$$

The steady-states may cross the threshold $\theta$ or $\Theta$ only. More interesting cases are that they may cross both thresholds $\theta$ and $\Theta$. Stable waves represent attractors of the dynamical system. We also investigate bifurcations of solutions of these equations. The kernel is either an even probability function with exponential decay at infinity or a Mexican hat type function, e.g. $K(x)=A \exp (-a|x|)-B \exp (-b|x|)$ and $K(x)=A \exp \left(-a x^{2}\right)-B \exp \left(-b x^{2}\right)$, where $A>B>0$ and $a>b>0$ are constants. The firing rate $H$ is the Heaviside step function.

\section{Contents}

1. Introduction 72

2. steady-state solutions $\quad 78$

3. Nonlinear stability and linear instability $\quad 87$

4. Bifurcations 96

5. Appendix 97

References $\quad 100$

1991 Mathematics Subject Classification. 35B35 35R10 45K05 92C20.

Key words and phrases. Nonlocal neuronal networks, integral-differential equations, steadystate solutions, existence and stability, bifurcation. 


\section{Introduction}

In this paper, we are concerned with the following nonlinear system of integraldifferential equations arising from nonlocal neuronal networks

$$
\begin{aligned}
& \frac{\partial u}{\partial t}+u+w=(\alpha-\gamma u) \int_{\mathbb{R}} K(x-y) H(u(y, t)-\theta) d y \\
& +(\beta-\delta u) \int_{\mathbb{R}} K(x-y) H(u(y, t)-\Theta) d y, u(x, 0)=u_{0}(x), \\
\frac{\partial w}{\partial t} & =\varepsilon(u-\tau w), w(x, 0)=w_{0}(x) .
\end{aligned}
$$

We focus on the existence and nonlinear exponential stability (linear exponential instability, respectively) of steady-state solutions of the system. We also investigate bifurcations of the solutions. To achieve our main goal, we use ideas in nonlinear analysis.

In this system, $u(x, t)$ represents the membrane potential of a neuron at position $x$ and time $t$, while $w$ represents the leaking current processes on a slow time scale. The system represents a one-dimensional chain of neurons with nonlocal synaptic coupling, with $\alpha-\gamma u$ and $\beta-\delta u$ being the coupling strength. The constants $\theta$ and $\Theta$ are the thresholds for synaptic coupling to be activated. The integral terms on the right-hand side of equation (1) account for excitatory connections between the cell at $x$ and other neurons in the network. See [3]-[6] for more biological backgrounds.

To derive the nonlocal model equation, first of all, we consider one-dimensional chain of discrete array of neurons. Each neuron may represent a relaxation oscillator. Moreover, every neuron receives strong contributions of membrane potentials from some neurons and weak contributions from other neurons: $[\alpha-\gamma u(x, t)] K(x-$ $\left.y_{j}\right) H\left(u\left(y_{j}, t\right)-\theta\right) \triangle y_{j}$, where $K\left(x-y_{j}\right)$ (representing a synapse weight) can be viewed as the probability that there is a synaptic connection between the nerve cell at $y_{j}$ and the cell at $x ; H\left(u\left(y_{j}, t\right)-\theta\right)=1$ if $u\left(y_{j}, t\right)>\theta$ and $H\left(u\left(y_{j}, t\right)-\theta\right)=0$ if $u\left(y_{j}, t\right)<\theta ; \alpha-\gamma u(x, t)$ denotes synaptic coupling strength; and $\triangle y_{j}=y_{j}-y_{j-1}$. Note that synapses are the principal sites where neurons communicate with each other. Most neurons communicate with each other at chemical synapses in which messages are transmitted by the release of chemicals called neurotransmitters from the presynaptic cells and detected by receptors on the postsynaptic cells. Now we find that this coupling is indeed of chemical synapses type. Summing up the contributions of membrane potentials from all other neurons to a single neuron would yield the Riemann sum: $[\alpha-\gamma u(x, t)] \sum_{j=1}^{N} K\left(x-y_{j}\right) H\left(u\left(y_{j}, t\right)-\theta\right) \triangle y_{j}$, where $N$ denotes the total number of neurons.

Secondly, note that there are about a trillion $\left(10^{12}\right)$ neurons and approximately a zillion $\left(10^{15}\right)$ synapses and a single neuron may receive inputs from about $10^{4}$ synapses. Therefore the number of neurons and their tree-like dendritic structure in even a small piece of cortex is so large that a natural approach to take in modeling layers of cortex is to consider a continuum of cells. That is to say, a cortical layer of nerve tissue can be regarded as a neuronal field that supports and propagates interacting patterns of excitation. Motivated by this fact, if we consider an ideal situation, namely, along the one-dimensional neural field, the neurons are continuously distributed (so that $N \rightarrow+\infty$ ), then we obtain a nonlocal term: 
$(\alpha-\gamma u) \int_{\mathbb{R}} K(x-y) H(u(y, t)-\theta) d y$. Thus, the equation (1) is of great interest in nonlocal neuronal networks.

We consider a different characterization of the gain function in which there is a low persistent activity rate and a high saturating rate. This case differs from previous works and myself, because the system has two firing rates and two nonlocal terms. Mathematical analysis of this problem is more challenging than before.

Suppose that the constants $\alpha>0, \beta>0, \gamma \geq 0, \delta \geq 0,0<\varepsilon \ll 1, \theta>0$, $\Theta>0$ and $\tau>0$ satisfy the following assumptions

$$
0<\theta<\frac{\alpha}{1+\gamma}<\Theta<\frac{\alpha+\beta}{1+\gamma+\delta}
$$

and

$$
0<\theta<\frac{\alpha \tau}{1+(1+\gamma) \tau}<\Theta<\frac{(\alpha+\beta) \tau}{1+(1+\gamma+\delta) \tau}
$$

The kernel function describes the network architecture and it is a sufficiently regular even probability measure with exponential decay at infinity. Let $H(x)=0$ for all $x<0$ and $H(x)=1$ for all $x>0$.

We display several well-known results of (1)-(2). The main results are stated and established in the next three sections. Consider the scalar integral-differential equation

$$
u_{t}+u=(\alpha-\beta u) \int_{\mathbb{R}} K(x-y) H(u(y, t)-\theta) d y,
$$

with $0<\theta<\frac{\alpha}{1+\beta}$. Traveling wave fronts (namely, $u(x, t)=U(z), U_{z} \neq 0$, where $z=x+\nu t$ and $\nu$ denotes wave-speed) are probably one of the most interesting solutions of (3). Waves with positive or negative wave-speed satisfy

$$
\nu U_{z}+U=(\alpha-\beta U) \int_{\mathbb{R}} K(z-y) H(U(y)-\theta) d y
$$

Theorem A. Let $\beta=0$. For each fixed kernel function satisfying $0 \leq K(x) \leq$ $C \exp (-\rho|x|), K(-x)=K(x)$ for all $x \in \mathbb{R}$ and $\int_{\mathbb{R}} K(x) d x=1$, where $C>0$ and $\rho>0$ are constants, there are two wave fronts, one increasing and the other decreasing, with one of the wave-speeds $\pm \nu_{0}$ :

$$
\begin{aligned}
U_{\text {increasing }}(z) & =\alpha \int_{-\infty}^{z} K(x) d x-\alpha \int_{-\infty}^{z} \exp \left(\frac{x-z}{\nu_{0}}\right) K(x) d x, \\
\frac{\alpha}{2}-\theta & =\alpha \int_{-\infty}^{0} \exp \left(+\frac{x}{\nu_{0}}\right) K(x) d x, z=x+\nu_{0} t \\
U_{\text {decreasing }}(z) & =\alpha \int_{z}^{\infty} K(x) d x-\alpha \int_{z}^{\infty} \exp \left(\frac{z-x}{\nu_{0}}\right) K(x) d x, \\
\frac{\alpha}{2}-\theta & =\alpha \int_{0}^{\infty} \exp \left(-\frac{x}{\nu_{0}}\right) K(x) d x, z=x-\nu_{0} t
\end{aligned}
$$


if $0<\theta<\frac{\alpha}{2}$, and very similarly,

$$
\begin{aligned}
U_{\text {decreasing }}(z) & =\alpha \int_{z}^{\infty} K(x) d x+\alpha \int_{-\infty}^{z} \exp \left(\frac{x-z}{\nu_{0}}\right) K(x) d x, \\
\theta-\frac{\alpha}{2} & =\alpha \int_{-\infty}^{0} \exp \left(+\frac{x}{\nu_{0}}\right) K(x) d x, z=x+\nu_{0} t, \\
U_{\text {increasing }}(z) & =\alpha \int_{-\infty}^{z} K(x) d x+\alpha \int_{z}^{\infty} \exp \left(\frac{z-x}{\nu_{0}}\right) K(x) d x, \\
\theta-\frac{\alpha}{2} & =\alpha \int_{0}^{\infty} \exp \left(-\frac{x}{\nu_{0}}\right) K(x) d x, z=x-\nu_{0} t,
\end{aligned}
$$

if $\theta>\frac{\alpha}{2}$. There are two steady-state solutions

$$
U_{\text {increasing }}(z)=\alpha \int_{-\infty}^{z} K(x) d x \text { and } U_{\text {decreasing }}(z)=\alpha \int_{z}^{\infty} K(x) d x,
$$

if $\theta=\frac{\alpha}{2}$. Overall, there hold $U(0)=\theta$. Either $U_{z} \geq 0$ on $\mathbb{R}$ and

$$
\lim _{z \rightarrow-\infty}\left(U(z), U_{z}(z)\right)=(0,0), \lim _{z \rightarrow+\infty}\left(U(z), U_{z}(z)\right)=(\alpha, 0),
$$

or $U_{z} \leq 0$ on $\mathbb{R}$ and

$$
\lim _{z \rightarrow-\infty}\left(U(z), U_{z}(z)\right)=(\alpha, 0), \lim _{z \rightarrow+\infty}\left(U(z), U_{z}(z)\right)=(0,0) .
$$

The wave-speed is a well defined function of $\frac{\theta}{\alpha}$, such that

$$
\lim _{\frac{\theta}{\alpha} \rightarrow 0^{+}} \nu\left(\frac{\theta}{\alpha}\right)=+\infty, \lim _{\frac{\theta}{\alpha} \rightarrow \frac{1}{2}} \nu\left(\frac{\theta}{\alpha}\right)=0^{+}, \lim _{\frac{\theta}{\alpha} \rightarrow 1^{-}} \nu\left(\frac{\theta}{\alpha}\right)=+\infty .
$$

Moreover

$$
\begin{aligned}
& \lim _{\frac{\theta}{\alpha} \rightarrow 0^{+}} U_{\text {increasing }}\left(z, \frac{\theta}{\alpha}\right)=\alpha H\left(\theta-\frac{\alpha}{2}\right), \\
& \lim _{\frac{\theta}{\alpha} \rightarrow \frac{1}{2}} U_{\text {increasing }}\left(z, \frac{\theta}{\alpha}\right)=\alpha \int_{-\infty}^{z} K(x) d x, \\
& \lim _{\frac{\theta}{\alpha} \rightarrow 1^{-}} U_{\text {increasing }}\left(z, \frac{\theta}{\alpha}\right)=\alpha H\left(\theta-\frac{\alpha}{2}\right),
\end{aligned}
$$

and

$$
\begin{aligned}
\lim _{\frac{\theta}{\alpha} \rightarrow 0^{+}} U_{\text {decreasing }}\left(z, \frac{\theta}{\alpha}\right) & =\alpha H\left(\theta-\frac{\alpha}{2}\right), \\
\lim _{\frac{\theta}{\alpha} \rightarrow \frac{1}{2}} U_{\text {decreasing }}\left(z, \frac{\theta}{\alpha}\right) & =\alpha \int_{z}^{\infty} K(x) d x, \\
\lim _{\frac{\theta}{\alpha} \rightarrow 1^{-}} U_{\text {decreasing }}\left(z, \frac{\theta}{\alpha}\right) & =\alpha H\left(\theta-\frac{\alpha}{2}\right) .
\end{aligned}
$$

Proof. See [13]-[15] for its proof.

Note that when $\varepsilon=0$, the second variable $w$ in system (1)-(2) becomes a constant. The equation

$$
\nu U_{z}+U+W=(\alpha-\beta U) \int_{\mathbb{R}} K(z-y) H(U(y)-\theta) d y,
$$


has a decreasing (increasing, respectively) traveling wave solution $U$, such that $U(0)=\theta$, if the counterpart equation (4) has an increasing (decreasing, respectively) solution for $0<\theta<\frac{\alpha}{2}\left(\theta>\frac{\alpha}{2}>0\right.$, respectively), where $\nu= \pm \nu_{0}$ and $W=\alpha-2 \theta$.

The stability of each of these monotone traveling waves is determined completely by the essential spectrum and eigenvalues of an associate linear differential operator $\mathcal{L}$, see [13]. Furthermore, the eigenvalues of the operator coincide with the zeros of a complex analytic function $\mathcal{E}(\lambda)$, which is called the eigenvalue function. The eigenvalue function is defined by

$$
\mathcal{E}_{+}(\lambda)=1-\int_{-\infty}^{0} \exp \left(+\frac{\lambda+1}{\nu_{0}} x\right) K(x) d x / \int_{-\infty}^{0} \exp \left(+\frac{x}{\nu_{0}}\right) K(x) d x,
$$

for traveling waves with a positive speed $+\nu_{0}$, and by

$$
\mathcal{E}_{-}(\lambda)=1-\int_{0}^{\infty} \exp \left(-\frac{\lambda+1}{\nu_{0}} x\right) K(x) d x / \int_{0}^{\infty} \exp \left(-\frac{x}{\nu_{0}}\right) K(x) d x
$$

for waves with a negative speed $-\nu_{0}$. Making a change of variable $x \rightarrow-x$, we find $\mathcal{E}_{+}(\lambda)=\mathcal{E}_{-}(\lambda)$. Rigorous mathematical analysis demonstrates that all of these monotone traveling wave solutions are stable relative to (3), see [13].

When $\varepsilon>0$, under certain conditions on the parameters $\alpha, \gamma$ and $\theta$, the system (1)-(2) can support both slow and fast homoclinic orbits as well as heteroclinic orbit.

Theorem B. Suppose that $0<2 \theta<\alpha, \beta=0,0<\frac{\alpha \gamma}{1+\gamma}<\theta$ and $0<$ $\varepsilon \ll 1$. Then, there exist a unique slow homoclinic orbit $\left(U_{\text {slow }}(\varepsilon, \cdot), W_{\text {slow }}(\varepsilon, \cdot)\right)$ corresponding to a unique slow wave-speed $\nu_{\text {slow }}(\varepsilon)$, and a unique fast homoclinic orbit $\left(U_{\text {fast }}(\varepsilon, \cdot), W_{\text {fast }}(\varepsilon, \cdot)\right)$ corresponding to a unique fast wave-speed $\nu_{\text {fast }}(\varepsilon)$. The slow wave-speed $\nu_{\text {slow }}(\varepsilon)=\kappa_{1}(\varepsilon) \varepsilon$ and the fast wave-speed $\nu_{\text {fast }}(\varepsilon)=\nu_{0}-\kappa_{2}(\varepsilon) \varepsilon$, where $\kappa_{i}(\varepsilon)>0$ are constants such that $\kappa_{i}(\varepsilon)>0$ for all $0 \leq \varepsilon \ll 1$. Both homoclinic orbits satisfy the traveling wave equations

$$
\begin{aligned}
\nu U_{z}+U+W & =\alpha \int_{\mathbb{R}} K(z-y) H(U(y)-\theta) d y, \\
\nu W_{z} & =\varepsilon(U-\gamma W),
\end{aligned}
$$

and the homogeneous Dirichlet boundary conditions

$$
\lim _{z \rightarrow \pm \infty}(U(\varepsilon, z), W(\varepsilon, z))=\lim _{z \rightarrow \pm \infty}\left(U_{z}(\varepsilon, z), W_{z}(\varepsilon, z)\right)=(0,0) .
$$

Furthermore, the slow orbit is unstable and the fast orbit is stable, in the sense of $L^{\infty}(\mathbb{R}) \times L^{\infty}(\mathbb{R})$-norm. Suppose that $0<2 \theta<\alpha, \frac{\alpha \gamma}{1+\gamma}>\theta$ and $0<\varepsilon \ll 1$. Then, there exists a unique heteroclinic orbit $\left(U_{\text {hetero }}(\varepsilon, \cdot), W_{\text {hetero }}(\varepsilon, \cdot)\right)$ corresponding to a unique wave-speed $\nu_{\text {hetero }}(\varepsilon)=\nu_{0}-\kappa(\varepsilon) \varepsilon$. The heteroclinic orbit satisfies the above traveling wave equations (5)-(6) and the Dirichlet boundary conditions

$$
\begin{gathered}
\lim _{z \rightarrow-\infty}(U(\varepsilon, z), W(\varepsilon, z))=(0,0), \lim _{z \rightarrow+\infty}(U(\varepsilon, z), W(\varepsilon, z))=\frac{\alpha}{1+\gamma}(\gamma, 1), \\
\lim _{z \rightarrow \pm \infty}\left(U_{z}(\varepsilon, z), W_{z}(\varepsilon, z)\right)=(0,0) .
\end{gathered}
$$

The heteroclinic orbit is also stable relative to system (1)-(2).

Proof. See [8], [13], [15] for its proof.

Remark 1. The slow homoclinic orbit being unstable and the fast homoclinic orbit being stable is consistent with the results of the nonlinear singularly perturbed system of Fitzhugh-Nagumo equations $u_{t}=u_{x x}+u(1-u)(u-a)-w, w_{t}=\varepsilon(u-\gamma w)$, 
where $0<a<\frac{1}{2}, \gamma>0$ and $0<\varepsilon \ll 1$ (this is a single cell cable model), see [10][11].

Consider the scalar integral-differential equation

$$
\begin{aligned}
& \frac{\partial u}{\partial t}+u=(\alpha-\gamma u) \int_{\mathbb{R}} K(x-y) H(u(y, t)-\theta) d y \\
& \quad+(\beta-\delta u) \int_{\mathbb{R}} K(x-y) H(u(y, t)-\Theta) d y .
\end{aligned}
$$

Traveling wave fronts (namely, $u(x, t)=U(z), U_{z} \neq 0$, where $z=x+\nu t$ and $\nu$ denotes wave-speed) are probably one of the most interesting solutions of (3) and they satisfy

$$
\begin{aligned}
& \nu U_{z}+U=(\alpha-\gamma U) \int_{\mathbb{R}} K(z-y) H(U(y)-\theta) d y \\
& \quad+(\beta-\delta U) \int_{\mathbb{R}} K(z-y) H(U(y)-\Theta) d y
\end{aligned}
$$

Theorem C. Suppose that $2 \theta<\alpha<\Theta<\alpha+\frac{\beta}{2}$ and $\gamma=\delta=0$. For each fixed kernel function satisfying $0 \leq K(x) \leq C \exp (-\rho|x|), K(-x)=K(x)$ for all $x \in \mathbb{R}$ and $\int_{\mathbb{R}} K(x) d x=1$, where $C>0$ and $\rho>0$ are constants, there are exactly three monotonically increasing, exponentially stable traveling wave fronts to equation (4). The first traveling wave front is given by

$$
\begin{aligned}
U_{1}(z) & =\alpha \int_{-\infty}^{z} K(x) d x-\alpha \int_{-\infty}^{z} \exp \left(\frac{x-z}{\nu_{1}}\right) K(x) d x \\
\frac{d}{d z} U_{1}(z) & =\frac{\alpha}{\nu_{1}} \int_{-\infty}^{z} \exp \left(\frac{x-z}{\nu_{1}}\right) K(x) d x \\
\lim _{z \rightarrow-\infty} U_{1}(z) & =0, \lim _{z \rightarrow+\infty} U_{1}(z)=\alpha, \lim _{z \rightarrow \pm \infty} \frac{d}{d z} U_{1}(z)=0
\end{aligned}
$$

and the first wave-speed $\nu=\nu_{1}>0$ is determined by

$$
\frac{\alpha}{2}-\alpha \int_{-\infty}^{0} \exp \left(\frac{x}{\nu_{1}}\right) K(x) d x=\theta
$$

The second front is

$$
\begin{aligned}
U_{2}(z) & =\alpha+\beta \int_{-\infty}^{z} K(x) d x-\beta \int_{-\infty}^{z} \exp \left(\frac{x-z}{\nu_{2}}\right) K(x) d x, \\
\frac{d}{d z} U_{2}(z) & =\frac{\beta}{\nu_{2}} \int_{-\infty}^{z} \exp \left(\frac{x-z}{\nu_{2}}\right) K(x) d x \\
\lim _{z \rightarrow-\infty} U_{2}(z) & =\alpha, \lim _{z \rightarrow+\infty} U_{2}(z)=\alpha+\beta, \lim _{z \rightarrow \pm \infty} \frac{d}{d z} U_{2}(z)=0,
\end{aligned}
$$

and the second wave-speed $\nu=\nu_{2}$ is determined by

$$
\alpha+\frac{\beta}{2}-\beta \int_{-\infty}^{0} \exp \left(\frac{x}{\nu_{2}}\right) K(x) d x=\Theta .
$$


The third front is

$$
\begin{aligned}
U_{3}(z)= & \alpha \int_{-\infty}^{z} K(x) d x+\beta \int_{-\infty}^{z-Z} K(x) d x \\
& -\int_{-\infty}^{z} \exp \left(\frac{x-z}{\nu_{3}}\right)[\alpha K(x)+\beta K(x-Z)] d x \\
\frac{d}{d z} U_{3}(z)= & \frac{1}{\nu_{3}} \int_{-\infty}^{z} \exp \left(\frac{x-z}{\nu_{3}}\right)[\alpha K(x)+\beta K(x-Z)] d x, \\
\lim _{z \rightarrow-\infty} U_{3}(z)= & 0, \lim _{z \rightarrow+\infty} U_{3}(z)=\alpha+\beta, \lim _{z \rightarrow \pm \infty} \frac{d}{d z} U_{3}(z)=0,
\end{aligned}
$$

and the third wave-speed $\nu=\nu_{3}$ together with the crossing $Z$ are determined by

$$
\frac{\alpha}{2}+\beta \int_{-\infty}^{-Z} K(x) d x-\int_{-\infty}^{0} \exp \left(\frac{x}{\nu_{3}}\right)[\alpha K(x)+\beta K(x-Z)] d x=\theta
$$

and

$$
\alpha \int_{-\infty}^{Z} K(x) d x+\frac{\beta}{2}-\int_{-\infty}^{0} \exp \left(\frac{x}{\nu_{3}}\right)[\alpha K(x+Z)+\beta K(x)] d x=\Theta .
$$

Proof. See [15] for its proof.

Note that when $\varepsilon=0$, the second variable $w$ in system (1)-(2) becomes a constant. The equation

$$
\begin{aligned}
\nu U_{z}+ & U+W=(\alpha-\gamma U) \int_{\mathbb{R}} K(z-y) H(U(y)-\theta) d y \\
& +(\beta-\delta U) \int_{\mathbb{R}} K(z-y) H(U(y)-\Theta) d y
\end{aligned}
$$

has three decreasing traveling wave solutions, such that $U_{1}(0)=\theta, U_{2}(0)=\Theta$, $U_{3}(0)=\theta$ and $U_{3}(S)=\Theta$, for some unique $S<0$.

The spectral stability of each of these monotone traveling waves is determined completely by the essential spectrum and eigenvalues of an associate linear differential operator $\mathcal{L}$, see [13]-[15]. Furthermore, the eigenvalues of the operator coincide with the zeros of a complex analytic function $\mathcal{E}(\lambda)$, called the eigenvalue function. Rigorous mathematical analysis demonstrates that all of these monotone traveling wave solutions are spectrally stable relative to (3), see [13]-[15].

When $\varepsilon>0$, under certain conditions on the parameters $\alpha, \beta, \gamma, \delta, \varepsilon, \theta, \Theta$ and $\tau$, the system (1)-(2) can support both slow and fast homoclinic orbits as well as heteroclinic orbit.

Theorem D. (I) There are three exponentially stable, homoclinic orbits. They satisfy the boundary conditions

$$
\begin{aligned}
\lim _{z \rightarrow \pm \infty}\left(U_{1}(\varepsilon, z), W_{1}(\varepsilon, z)\right) & =(0,0), \\
\lim _{z \rightarrow \pm \infty}\left(U_{2}(\varepsilon, z), W_{2}(\varepsilon, z)\right) & =\left(\frac{\alpha \gamma}{1+\gamma}, \frac{\alpha}{1+\gamma}\right), \\
\lim _{z \rightarrow \pm \infty}\left(U_{2}(\varepsilon, z), W_{2}(\varepsilon, z)\right) & =(0,0) .
\end{aligned}
$$

Furthermore, $\left(U_{1}, W_{1}\right)$ crosses the threshold $\theta$ twice but does not cross $\Theta,\left(U_{2}, W_{2}\right)$ crosses the threshold $\Theta$ twice but does not cross $\theta$, and $\left(U_{3}, W_{3}\right)$ crosses the threshold 
$\theta$ twice and the threshold $\Theta$ twice. (II) There are precisely three exponentially stable, heteroclinic orbits. They satisfy the following boundary conditions

$$
\begin{aligned}
& \lim _{z \rightarrow-\infty}\left(\mathbb{U}_{1}(\varepsilon, z), \mathbb{W}_{1}(\varepsilon, z)\right)=(0,0), \\
& \lim _{z \rightarrow+\infty}\left(\mathbb{U}_{1}(\varepsilon, z), \mathbb{W}_{1}(\varepsilon, z)\right)=\left(\frac{\alpha \gamma}{1+\gamma}, \frac{\alpha}{1+\gamma}\right), \\
& \lim _{z \rightarrow-\infty}\left(\mathbb{U}_{2}(\varepsilon, z), \mathbb{W}_{2}(\varepsilon, z)\right)=\left(\frac{\alpha \gamma}{1+\gamma}, \frac{\alpha}{1+\gamma}\right), \\
& \lim _{z \rightarrow+\infty}\left(\mathbb{U}_{2}(\varepsilon, z), \mathbb{W}_{2}(\varepsilon, z)\right)=\left(\frac{\alpha \gamma+\beta \gamma}{1+\gamma}, \frac{\alpha+\beta}{1+\gamma}\right), \\
& \lim _{z \rightarrow-\infty}\left(\mathbb{U}_{3}(\varepsilon, z), \mathbb{W}_{3}(\varepsilon, z)\right)=(0,0), \\
& \lim _{z \rightarrow+\infty}\left(\mathbb{U}_{3}(\varepsilon, z), \mathbb{W}_{3}(\varepsilon, z)\right)=\left(\frac{\alpha \gamma+\beta \gamma}{1+\gamma}, \frac{\alpha+\beta}{1+\gamma}\right) .
\end{aligned}
$$

Furthermore, the second component of each of the heteroclinic orbits is strictly increasing on $\mathbb{R}$. $\left(\mathbb{U}_{1}, \mathbb{W}_{1}\right)$ crosses the threshold $\theta$ once but does not cross $\Theta,\left(\mathbb{U}_{2}, \mathbb{W}_{2}\right)$ crosses the threshold $\Theta$ once but does not cross $\theta$ and $\left(\mathbb{U}_{3}, \mathbb{W}_{3}\right)$ crosses the threshold $\theta$ once and the threshold $\Theta$ once.

Proof. See [15] for its proof.

Remark. There are three exponentially unstable, homoclinic orbits with the same boundary conditions and crossings as above.

\section{2. steady-state solutions}

Suppose that either the kernel function is nonnegative, even, at least piecewise smooth, such that

$$
\int_{\mathbb{R}} K(x) d x=1, K^{\prime}(x) \leq 0 \text { in } \mathbb{R}^{+}, K(x) \leq C \exp (-\rho|x|) \text { in } \mathbb{R},
$$

for some positive constants $C$ and $\rho$; or the kernel is a Mexican hat type function, such as $K(x)=A \exp (-a|x|)-B \exp (-b|x|)$ and $K(x)=A \exp \left(-a x^{2}\right)-$ $B \exp \left(-b x^{2}\right)$, where $A>B>0$ and $a>b>0$ are constants, such that $\int_{\mathbb{R}} K(x) d x=$ $1, K^{\prime}(x)<0$ on $(0, M)$ and $K^{\prime}(x)>0$ on $(M,+\infty)$, for some constant $M>0$. In neuroscience, lateral inhibition (i.e. short distance connection represent excitatory connections and long range connections represent inhibitory connections) is of great interests. The corresponding kernel functions look like Mexican hats.

We focus on the analysis with nonnegative kernel functions. The steady-states are always positive if the kernel is positive and some of them are monotone. But for Mexican hat type kernels, the steady-states are not necessarily positive or monotone.

2.1. Existence and spectral instability: the case $\varepsilon=0$. We study nontrivial steady-states of the scalar equation (8) in this subsection.

Theorem 1. (I) When $(2+\gamma) \theta<\alpha$, there exists a unique positive steady-state $U$, such that $U(0)=U\left(X_{*}\right)=\theta, U<\theta$ on $(-\infty, 0) \bigcup\left(X_{*},+\infty\right)$ and $\theta<U<\Theta$ on $\left(0, X_{*}\right)$, for some $X_{*}>0$. (II) When $(2+\gamma) \theta=\alpha$, there are exactly two positive, monotone steady-states $U$, such that $U(0)=\theta$. (III) When $(2+\gamma) \theta>\alpha$, there exists a unique positive steady-state $U$, such that $U(0)=U\left(X^{*}\right)=\theta, \theta<U<\Theta$ 
on $(-\infty, 0) \bigcup\left(X^{*},+\infty\right)$ and $U<\theta$ on $\left(0, X^{*}\right)$ for some $X^{*}>0$. (IV) The steadystates in the first and the third cases are spectrally unstable relative to equation (8).

Proof. The steady-state solutions should satisfy the equation

$$
U(x)=(\alpha-\gamma U) \int_{\mathbb{R}} K(x-y) H(U(y)-\theta) d y .
$$

(I) Let $(2+\gamma) \theta<\alpha$. By using mean value theorem, it is straightforward to show that there exists a unique number $X_{*}>0$, such that

$$
\alpha \int_{0}^{X_{*}} K(x) d x /\left[1+\gamma \int_{0}^{X_{*}} K(x) d x\right]=\theta .
$$

The steady-state solution is given by

$$
U(x)=\alpha \int_{0}^{X_{*}} K(x-y) d y /\left[1+\gamma \int_{0}^{X_{*}} K(x-y) d y\right]
$$

Furthermore $K^{\prime} \leq 0$ on $\mathbb{R}^{+}$implies that $U^{\prime} \geq 0$ on $\left(-\infty, \frac{1}{2} X_{*}\right)$ and $U^{\prime} \leq 0$ on $\left(\frac{1}{2} X_{*},+\infty\right)$, thus $U(0)=U\left(X_{*}\right)=\theta, U<\theta$ on $(-\infty, 0) \bigcup\left(X_{*},+\infty\right)$ and $\theta<U<\Theta$ on $\left(0, X_{*}\right)$. Therefore, $U$ is the desired steady-state. Additionally, there hold

$$
\begin{aligned}
U^{\prime}(x) & =\alpha\left[K(x)-K\left(x-X_{*}\right)\right] /\left[1+\gamma \int_{0}^{X_{*}} K(x-y) d y\right]^{2}, \\
U^{\prime}(0) & =\alpha\left[K(0)-K\left(X_{*}\right)\right] /\left[1+\gamma \int_{0}^{X_{*}} K(x) d x\right]^{2}, \\
U^{\prime}\left(X_{*}\right) & =\alpha\left[K\left(X_{*}\right)-K(0)\right] /\left[1+\gamma \int_{0}^{X_{*}} K(x) d x\right]^{2}, \\
\left|U^{\prime}(0)\right| & =\left|U^{\prime}\left(X_{*}\right)\right|=\frac{1}{\alpha}(\alpha-\gamma \theta)^{2}\left[K(0)-K\left(X_{*}\right)\right] .
\end{aligned}
$$

The steady-state is symmetric about the $x=\frac{X_{*}}{2}$-axis, because

$$
\begin{aligned}
& U\left(\frac{X_{*}}{2}-x\right) \\
= & \alpha \int_{0}^{X_{*}} K\left(\frac{X_{*}}{2}-x-y\right) d y /\left[1+\gamma \int_{0}^{X_{*}} K\left(\frac{X_{*}}{2}-x-y\right) d y\right] \\
= & \alpha \int_{0}^{X_{*}} K\left(\frac{X_{*}}{2}+x-\zeta\right) d \zeta /\left[1+\gamma \int_{0}^{X_{*}} K\left(\frac{X_{*}}{2}+x-\zeta\right) d \zeta\right] \\
= & U\left(x+\frac{X_{*}}{2}\right)
\end{aligned}
$$

where we have made a change of variable $\zeta=X_{*}-y$ and also applied the property $K(-x)=K(x)$ for all $x$. The uniqueness of the steady-state (up to translation invariance) is obviously true. Due to the assumption $|K(x)| \leq C \exp (-\rho|x|)$ on $\mathbb{R}$, the steady-state converges to zero exponentially fast as $z \rightarrow \pm \infty$. 
(II) Let $(2+\gamma) \theta=\alpha$, then

$$
\begin{aligned}
U_{\text {increasing }}(x) & =\alpha \int_{-\infty}^{x} K(\xi) d \xi /\left[1+\gamma \int_{-\infty}^{x} K(\xi) d \xi\right], \\
U_{\text {decreasing }}(x) & =\alpha \int_{x}^{\infty} K(\xi) d \xi /\left[1+\gamma \int_{x}^{\infty} K(\xi) d \xi\right],
\end{aligned}
$$

are the steady-states of (8). Moreover

$$
\begin{aligned}
U_{\text {increasing }}^{\prime}(x) & =+\alpha K(x) /\left[1+\gamma \int_{-\infty}^{x} K(\xi) d \xi\right]^{2}, \\
U_{\text {decreasing }}^{\prime}(x) & =-\alpha K(x) /\left[1+\gamma \int_{x}^{\infty} K(\xi) d \xi\right]^{2}, \\
\left|U_{\text {increasing }}^{\prime}(0)\right| & =\left|U_{\text {decreasing }}^{\prime}(0)\right|=\frac{4}{\alpha} K(0) \theta^{2} .
\end{aligned}
$$

(III) Let $(2+\gamma) \theta>\alpha>(1+\gamma) \theta$, then very similar to the case $(2+\gamma) \theta<\alpha$, there exists a positive number $X^{*}$ satisfying

$$
\left[\alpha-\alpha \int_{0}^{X^{*}} K(x) d x\right] /\left[1+\gamma-\gamma \int_{0}^{X^{*}} K(x) d x\right]=\theta .
$$

There is a unique nontrivial steady-state

$$
U(x)=\left[\alpha-\alpha \int_{0}^{X^{*}} K(x-y) d y\right] /\left[1+\gamma-\gamma \int_{0}^{X^{*}} K(x-y) d y\right] .
$$

Moreover

$$
\begin{aligned}
U^{\prime}(x) & =\alpha\left[K\left(x-X^{*}\right)-K(x)\right] /\left[1+\gamma-\gamma \int_{0}^{X^{*}} K(x-y) d y\right]^{2}, \\
U^{\prime}(0) & =\alpha\left[K\left(X^{*}\right)-K(0)\right] /\left[1+\gamma-\gamma \int_{0}^{X^{*}} K(x) d x\right]^{2}, \\
U^{\prime}\left(X^{*}\right) & =\alpha\left[K(0)-K\left(X^{*}\right)\right] /\left[1+\gamma-\gamma \int_{0}^{X^{*}} K(x) d x\right]^{2}, \\
\left|U^{\prime}(0)\right| & =\left|U^{\prime}\left(X^{*}\right)\right|=\frac{1}{\alpha}(\alpha-\gamma \theta)^{2}\left[K(0)-K\left(X^{*}\right)\right] .
\end{aligned}
$$

(IV) Let $(2+\gamma) \theta \neq \alpha$ so that either $X_{*}$ or $X^{*}$ is well-defined. The case $(2+\gamma) \theta=\alpha$ can be investigated similarly. The linearization of (8) about one of the steady-states $U$ is

$$
\begin{aligned}
& P_{t}(x, t)+\left[1+\gamma \int_{\mathbb{R}} K(x-y) H(U(y)-\theta) d y\right] P(x, t) \\
= & \frac{\alpha-\gamma U(x)}{\left|U^{\prime}(0)\right|} K(x) P(0, t)+\frac{\alpha-\gamma U(x)}{\left|U^{\prime}(X)\right|} K(x-X) P(X, t),
\end{aligned}
$$

where $X=X_{*}$ or $X=X^{*}$, and the corresponding eigenvalue problem is

$$
\begin{aligned}
& {\left[\lambda+1+\gamma \int_{\mathbb{R}} K(x-y) H(U(y)-\theta) d y\right] \psi(x) } \\
= & \frac{\alpha-\gamma U(x)}{\left|U^{\prime}(0)\right|} K(x) \psi(0)+\frac{\alpha-\gamma U(x)}{\left|U^{\prime}(X)\right|} K(x-X) \psi(X) .
\end{aligned}
$$


Define the open, unbounded region $\Omega=\{\lambda \in \mathbb{C}$ : $\operatorname{Re} \lambda>-1\}$. We will study the eigenvalue problem in $\Omega$. If $\psi(0)=\psi(X)=0$, then $\psi \equiv 0$. If $\psi(0)=0$ or $\psi(X)=0$, then simple analysis also shows that $\psi \equiv 0$. Without loss of generality, we assume that $\psi(0) \neq 0$ and $\psi(X) \neq 0$. Now we define the linear operator $\mathcal{L}: C^{0}(\mathbb{R}) \bigcap L^{\infty}(\mathbb{R}) \rightarrow C^{0}(\mathbb{R}) \bigcap L^{\infty}(\mathbb{R})$ by

$$
\begin{gathered}
\mathcal{L} \psi=-\left[1+\gamma \int_{\mathbb{R}} K(x-y) H(U(y)-\theta) d y\right] \psi \\
+\frac{\alpha-\gamma U(x)}{\left|U^{\prime}(0)\right|} K(x) \psi(0)+\frac{\alpha-\gamma U(x)}{\left|U^{\prime}(X)\right|} K(x-X) \psi(X) .
\end{gathered}
$$

The essential spectrum of $\mathcal{L}$ consists of those numbers satisfying $\operatorname{Re} \lambda=-1$. Due to translation invariance of the steady-states, $\lambda=0$ is an eigenvalue of $\mathcal{L}$ and $U^{\prime}$ is the corresponding eigenfunction. To find other eigenvalues, setting $x=0$ and $x=X$ in the eigenvalue problems, we get

$$
\left(\lambda+\frac{\alpha}{\alpha-\gamma \theta}-\frac{\alpha-\gamma \theta}{\left|U^{\prime}(0)\right|} K(0)\right) \psi(0)=\frac{\alpha-\gamma \theta}{\left|U^{\prime}(X)\right|} K(X) \psi(X),
$$

and

Therefore

$$
\left(\lambda+\frac{\alpha}{\alpha-\gamma \theta}-\frac{\alpha-\gamma \theta}{\left|U^{\prime}(X)\right|} K(0)\right) \psi(X)=\frac{\alpha-\gamma \theta}{\left|U^{\prime}(0)\right|} K(X) \psi(0) .
$$

$$
\begin{gathered}
{\left[\lambda+\frac{\alpha}{\alpha-\gamma \theta}-\frac{\alpha-\gamma \theta}{\left|U^{\prime}(0)\right|} K(0)\right]\left[\lambda+\frac{\alpha}{\alpha-\gamma \theta}-\frac{\alpha-\gamma \theta}{\left|U^{\prime}(X)\right|} K(0)\right]} \\
=\frac{[(\alpha-\gamma \theta) K(X)]^{2}}{\left|U^{\prime}(0)\right|\left|U^{\prime}(X)\right|} .
\end{gathered}
$$

Note that $\left|U^{\prime}(0)\right|=\left|U^{\prime}(X)\right|$ if $(2+\gamma) \theta \neq \alpha$. Solving this algebraic equation, we find that

and

$$
\lambda_{1}=(\alpha-\gamma \theta) \frac{K(0)-K(X)}{\left|U^{\prime}(0)\right|}-\frac{\alpha}{\alpha-\gamma \theta}=0,
$$

$$
\lambda_{2}=(\alpha-\gamma \theta) \frac{K(0)+K(X)}{\left|U^{\prime}(0)\right|}-\frac{\alpha}{\alpha-\gamma \theta}>0 .
$$

Therefore, any of the steady-states is spectrally unstable.

Suppose that $(2+\gamma) \theta=\alpha$. Then by similar analysis, we can show that there exists no nonzero eigenvalue of the associated linear operator $\mathcal{L}$ in the right half plane $\{\lambda: \operatorname{Re} \lambda>-1\}$, and the number $\lambda=0$ is a simple eigenvalue of $\mathcal{L}$. Thus the monotone steady-states are spectrally stable relative to (8).

Remark . If $(2+\gamma) \theta \neq \alpha$, then there are two eigenvalues, counting algebraic multiplicities, because the steady-state crosses the threshold $\theta$ twice. If $(2+\gamma) \theta=\alpha$, then there is only one eigenvalue, also counting algebraic multiplicity, because the steady-state crosses the threshold $\theta$ only once.

Theorem 2. (I) When $(2+2 \gamma+\delta) \Theta<2 \alpha+\beta$, there exists a unique positive steady-state $U$, such that $U(0)=U\left(Y_{*}\right)=\Theta, \theta<U<\Theta$ on $(-\infty, 0) \bigcup\left(Y_{*},+\infty\right)$ and $U>\Theta$ on $\left(0, Y_{*}\right)$, for some $Y_{*}>0$. (II) When $(2+2 \gamma+\delta) \Theta=2 \alpha+\beta$, there are exactly two positive, monotone steady-states $U$, such that $U(0)=\Theta$. (III) When $(2+2 \gamma+\delta) \Theta>2 \alpha+\beta$, there exists a unique positive steady-state $U$, such that 
$U(0)=U\left(Y^{*}\right)=\Theta, U>\Theta$ on $(-\infty, 0) \bigcup\left(Y^{*},+\infty\right)$ and $\theta<U<\Theta$ on $\left(0, Y^{*}\right)$ for some $Y^{*}>0$. (IV) The steady-states in the first and the third cases are spectrally unstable relative to equation (8).

Proof. It is very similar to that of Theorem 1 and is omitted. \#

Theorem 3. Suppose that $(\alpha-\gamma \Theta)[(2+\delta) \Theta-\beta]>0$ and $(\beta-\delta \theta)[(2+\gamma) \theta-\alpha]>$ 0 , such that

$$
\frac{(2+\gamma) \theta-\alpha}{2(\beta-\delta \theta)}+\frac{(2+\delta) \Theta-\beta}{2(\alpha-\gamma \Theta)}<1,
$$

for case (I),

$$
\frac{(2+\gamma) \theta-\alpha}{2(\beta-\delta \theta)}+\frac{(2+\delta) \Theta-\beta}{2(\alpha-\gamma \Theta)}=1
$$

for case (II), and

$$
\frac{(2+\gamma) \theta-\alpha}{2(\beta-\delta \theta)}+\frac{(2+\delta) \Theta-\beta}{2(\alpha-\gamma \Theta)}>1
$$

for case (III). (I) There exists a unique positive steady-state $U$, such that $U(0)=$ $U\left(Z_{*}\right)=\theta, U<\theta$ on $(-\infty, 0) \bigcup\left(Z_{*},+\infty\right)$ and $U>\theta$ on $\left(0, Z_{*}\right)$, for some $Z_{*}>0$; $U\left(\Gamma_{*}\right)=U\left(\Lambda_{*}\right)=\Theta, U<\Theta$ on $\left(-\infty, \Gamma_{*}\right) \bigcup\left(\Lambda_{*},+\infty\right)$ and $U>\Theta$ on $\left(\Gamma_{*}, \Lambda_{*}\right)$, for some constants $0<\Gamma_{*}<\Lambda_{*}<Z_{*}$. (II) There are exactly two positive, monotone steady-states $U$, such that $U(0)=\theta$ and $U\left(\Omega_{*}\right)=\Theta$ or $U(0)=\theta$ and $U\left(\Omega^{*}\right)=\Theta$, for some real numbers $\Omega_{*}$ and $\Omega^{*}$. (III) There exists a unique positive steady-state $U$, such that $U(0)=U\left(Z^{*}\right)=\theta, U>\theta$ on $(-\infty, 0) \bigcup\left(Z^{*},+\infty\right)$ and $U<\theta$ on $\left(0, Z^{*}\right)$ for some $Z^{*}>0 ; U\left(\Gamma^{*}\right)=U\left(\Lambda^{*}\right)=\Theta, U>\Theta$ on $\left(-\infty, \Gamma^{*}\right) \bigcup\left(\Lambda^{*},+\infty\right)$ and $U<\Theta$ on $\left(\Gamma^{*}, \Lambda^{*}\right)$, for some constants $\Gamma^{*}<0<Z^{*}<\Lambda^{*}$. (IV) The steadystates in the first and the third cases are spectrally unstable relative to equation (8).

Proof. (I) There exist three numbers $0<\Gamma_{*}<\Lambda_{*}<Z_{*}$, such that

$$
\begin{aligned}
(\alpha-\gamma \theta) \int_{0}^{Z_{*}} K(x) d x+(\beta-\delta \theta) \int_{\Gamma_{*}}^{\Lambda_{*}} K(x) d x & =\theta, \\
(\alpha-\gamma \Theta) \int_{\Lambda_{*}-Z_{*}}^{\Lambda_{*}} K(x) d x+(\beta-\delta \Theta) \int_{0}^{\Lambda_{*}-\Gamma_{*}} K(x) d x & =\Theta .
\end{aligned}
$$

The unique positive steady-state is given by

The derivative is

$$
\begin{gathered}
U(x)=\left\{\alpha \int_{x-Z_{*}}^{x} K(\xi) d \xi+\beta \int_{x-\Lambda_{*}}^{x-\Gamma_{*}} K(\xi) d \xi\right\} \\
/\left\{1+\gamma \int_{x-Z_{*}}^{x} K(\xi) d \xi+\delta \int_{x-\Lambda_{*}}^{x-\Gamma_{*}} K(\xi) d \xi\right\}
\end{gathered}
$$

$$
\begin{aligned}
& U^{\prime}(x)=\left\{\left[\alpha+(\alpha \delta-\beta \gamma) \int_{\Gamma_{*}}^{\Lambda_{*}} K(x-y) d y\right]\left[K(x)-K\left(x-Z_{*}\right)\right]\right. \\
&\left.+\left[\beta+(\beta \gamma-\alpha \delta) \int_{0}^{Z_{*}} K(x-y) d y\right]\left[K\left(x-\Lambda_{*}\right)-K\left(x-\Gamma_{*}\right)\right]\right\} \\
& \\
& /\left\{1+\gamma \int_{x-Z_{*}}^{x} K(\xi) d \xi+\delta \int_{x-\Lambda_{*}}^{x-\Gamma_{*}} K(\xi) d \xi\right\}^{2}
\end{aligned}
$$


(II) There exist exactly two real numbers $\Omega_{*}$ and $\Omega^{*}$, such that

$$
\begin{aligned}
& \frac{(2+\gamma) \theta-\alpha}{2(\beta-\delta \theta)}=\int_{-\infty}^{-\Omega_{*}} K(x) d x, \\
& \frac{(2+\delta) \Theta-\beta}{2(\alpha-\gamma \Theta)}=\int_{-\infty}^{\Omega_{*}} K(x) d x,
\end{aligned}
$$

and

$$
\begin{aligned}
\frac{(2+\gamma) \theta-\alpha}{2(\beta-\delta \theta)} & =\int_{-\Omega^{*}}^{\infty} K(x) d x, \\
\frac{(2+\delta) \Theta-\beta}{2(\alpha-\gamma \Theta)} & =\int_{\Omega^{*}}^{\infty} K(x) d x .
\end{aligned}
$$

The positive monotone steady-states are given by, respectively

$$
\begin{gathered}
U(x)=\left\{\alpha \int_{-\infty}^{x} K(\xi) d \xi+\beta \int_{-\infty}^{x-\Omega_{*}} K(\xi) d \xi\right\} \\
/\left\{1+\gamma \int_{-\infty}^{x} K(\xi) d \xi+\delta \int_{-\infty}^{x-\Omega_{*}} K(\xi) d \xi\right\}
\end{gathered}
$$

and

$$
\begin{gathered}
U(x)=\left\{\alpha \int_{x}^{\infty} K(\xi) d \xi+\beta \int_{x-\Omega^{*}}^{\infty} K(\xi) d \xi\right\} \\
/\left\{1+\gamma \int_{x}^{\infty} K(\xi) d \xi+\delta \int_{x-\Omega^{*}}^{\infty} K(\xi) d \xi\right\}
\end{gathered}
$$

The derivatives are

$$
\begin{aligned}
U^{\prime}(x)= & \left\{\left(\beta+(\beta \gamma-\alpha \delta) \int_{-\infty}^{x} K(\xi) d \xi\right) K\left(x-\Omega_{*}\right)\right. \\
& \left.+\left(\alpha+(\alpha \delta-\beta \gamma) \int_{-\infty}^{x-\Omega_{*}} K(\xi) d \xi\right) K(x)\right\} \\
& /\left\{1+\gamma \int_{-\infty}^{x} K(\xi) d \xi+\delta \int_{-\infty}^{x-\Omega_{*}} K(\xi) d \xi\right\}^{2},
\end{aligned}
$$

and

$$
\begin{aligned}
U^{\prime}(x)= & -\left\{\left(\beta+(\beta \gamma-\alpha \delta) \int_{x}^{\infty} K(\xi) d \xi\right) K\left(x-\Omega^{*}\right)\right. \\
& \left.+\left(\alpha+(\alpha \delta-\beta \gamma) \int_{x-\Omega_{*}}^{\infty} K(\xi) d \xi\right) K(x)\right\} \\
& /\left\{1+\gamma \int_{x}^{\infty} K(\xi) d \xi+\delta \int_{x-\Omega_{*}}^{\infty} K(\xi) d \xi\right\}^{2} .
\end{aligned}
$$

(III) There exist $\Gamma^{*}$ and $\Lambda^{*}$, such that

$$
\begin{aligned}
(\alpha-\gamma \theta)\left[1-\int_{0}^{Z^{*}} K(x) d x\right]+(\beta-\delta \theta)\left[1-\int_{\Gamma^{*}}^{\Lambda^{*}} K(x) d x\right] & =\theta, \\
(\alpha-\gamma \Theta)\left[1-\int_{\Lambda^{*}-Z^{*}}^{\Lambda^{*}} K(x) d x\right]+(\beta-\delta \Theta)\left[1-\int_{0}^{\Lambda^{*}-\Gamma^{*}} K(x) d x\right] & =\Theta .
\end{aligned}
$$


The unique positive steady-state is given by

$$
\begin{gathered}
U(x)=\left\{\alpha-\alpha \int_{x-Z^{*}}^{x} K(\xi) d \xi+\beta-\beta \int_{x-\Lambda^{*}}^{x-\Gamma^{*}} K(\xi) d \xi\right\} \\
/\left\{1+\gamma-\gamma \int_{x-Z^{*}}^{x} K(\xi) d \xi+\delta-\delta \int_{x-\Lambda^{*}}^{x-\Gamma^{*}} K(\xi) d \xi\right\}
\end{gathered}
$$

The derivative is

$$
\begin{aligned}
U^{\prime}(x)= & -\left\{\left[\alpha+(\alpha \delta-\beta \gamma)\left(1-\int_{x-\Lambda^{*}}^{x-\Gamma^{*}} K(\xi) d \xi\right)\right]\left[K(x)-K\left(x-Z^{*}\right)\right]\right. \\
+[\beta+ & \left.\left.(\beta \gamma-\alpha \delta)\left(1-\int_{x-Z^{*}}^{x} K(\xi) d \xi\right)\right]\left[K\left(x-\Lambda^{*}\right)-K\left(x-\Gamma^{*}\right)\right]\right\} \\
& /\left\{1+\gamma-\gamma \int_{x-Z^{*}}^{x} K(\xi) d \xi+\delta-\delta \int_{x-\Lambda^{*}}^{x-\Gamma^{*}} K(\xi) d \xi\right\}^{2} .
\end{aligned}
$$

It is very similar to that of Theorem 1 and is omitted.

2.2. Existence and spectral instability: the case $\varepsilon>0$. The parameter $\varepsilon>0$ is arbitrarily large in this subsection.

Theorem 4. Let $\theta<\frac{\alpha \tau}{1+(1+\gamma) \tau}<\Theta$. Then, there exist at least four nontrivial steady-state solutions to the equations (1)-(2). The steady-states are spectrally unstable relative to (1)-(2), if $(2+2 \tau+\gamma \tau) \theta \neq \alpha \tau$.

Proof. Solving the system

$$
\begin{aligned}
U(x)+W(x) & =(\alpha-\gamma U) \int_{\mathbb{R}} K(x-y) H(U(y)-\theta) d y, \\
U(x) & =\tau W(x),
\end{aligned}
$$

is equivalent to solving the system

$$
\begin{aligned}
U(x) & =\frac{\tau}{1+\tau}(\alpha-\gamma U) \int_{\mathbb{R}} K(x-y) H(U(y)-\theta) d y, \\
W(x) & =\frac{1}{1+\tau}(\alpha-\gamma U) \int_{\mathbb{R}} K(x-y) H(U(y)-\theta) d y .
\end{aligned}
$$

The rest of the analysis for the existence of the steady-states is the same as above (i.e the case $\varepsilon=0)$. If $(2+2 \tau+\gamma \tau) \theta<\alpha \tau$, then there exists a unique positive number $A_{*}$, such that

$$
\alpha \tau \int_{0}^{A_{*}} K(x) d x /\left[1+\tau+\gamma \tau \int_{0}^{A_{*}} K(x) d x\right]=\theta .
$$

The unique nontrivial steady-state is given by

$$
=\left\{\alpha \int_{0}^{A_{*}} K(x-y) d y /\left[1+\tau+\gamma \tau \int_{0}^{A_{*}} K(x-y) d y\right]\right\}(\tau, 1),
$$

and

$$
\left(U^{\prime}(x), W^{\prime}(x)\right)
$$




$$
=\left\{\alpha(1+\tau)\left[K(x)-K\left(x-A_{*}\right)\right] /\left[1+\tau+\gamma \tau \int_{0}^{A_{*}} K(x-y) d y\right]^{2}\right\}(\tau, 1) .
$$

Furthermore

$$
U^{\prime}(0)=\frac{(\alpha-\gamma \theta)^{2}}{\alpha(1+\tau)}\left[K(0)-K\left(A_{*}\right)\right]=-U^{\prime}\left(A_{*}\right)
$$

If $(2+2 \tau+\gamma \tau) \theta>\alpha \tau$, then there exists a unique positive number $A^{*}$, such that

$$
\left[\alpha \tau-\alpha \tau \int_{0}^{A^{*}} K(x) d x\right] /\left[1+\tau+\gamma \tau-\gamma \tau \int_{0}^{A^{*}} K(x) d x\right]=\theta .
$$

The unique nontrivial steady-state is given by

$$
=\left\{\left[\alpha-\alpha \int_{0}^{A^{*}} K(x-y) d y\right] /\left[1+\tau+\gamma \tau-\gamma \tau \int_{0}^{A^{*}} K(x-y) d y\right]\right\}(\tau, 1),
$$

and

$$
=\left\{\alpha(1+\tau)\left[K\left(x-A^{*}\right)-K(x)\right] /\left[1+\tau+\gamma \tau-\gamma \tau \int_{0}^{A^{*}} K(x-y) d y\right]^{2}\right\}(\tau, 1) .
$$

Moreover

$$
U^{\prime}(0)=\frac{(\alpha-\gamma \theta)^{2}}{\alpha(1+\tau)}\left[K\left(A^{*}\right)-K(0)\right]=-U^{\prime}\left(A^{*}\right) .
$$

If $(2+2 \tau+\gamma \tau) \theta=\alpha \tau$, then there are precisely two monotone steady-states: (i)

$$
\begin{gathered}
\left(U_{\text {increasing }}(x), W_{\text {increasing }}(x)\right) \\
=\left\{\alpha \int_{-\infty}^{x} K(\xi) d \xi /\left[1+\tau+\gamma \tau \int_{-\infty}^{x} K(\xi) d \xi\right]\right\}(\tau, 1),
\end{gathered}
$$

and the derivative

$$
\begin{gathered}
\left(U_{\text {increasing }}^{\prime}(x), W_{\text {increasing }}^{\prime}(x)\right) \\
=\left\{\alpha(1+\tau) K(x) /\left[1+\tau+\gamma \tau \int_{-\infty}^{x} K(\xi) d \xi\right]^{2}\right\}(\tau, 1),
\end{gathered}
$$

and (ii)

$$
=\left\{\alpha \int_{x}^{\infty} K(\xi) d \xi /\left[1+\tau+\gamma \tau \int_{x}^{\infty} K(\xi) d \xi\right]\right\}(\tau, 1),
$$

and the derivative

$$
=\left\{\begin{array}{c}
\left(U_{\text {decreasing }}^{\prime}(x), W_{\text {decreasing }}^{\prime}(x)\right) \\
\left.=\alpha(1+\tau) K(x) /\left[1+\tau+\gamma \tau \int_{x}^{\infty} K(\xi) d \xi\right]^{2}\right\}(\tau, 1) .
\end{array}\right.
$$

Now we obtain

$$
\left|U_{\text {increasing }}^{\prime}(0)\right|=\left|U_{\text {decreasing }}^{\prime}(0)\right|=\frac{4(1+\tau) \theta^{2}}{\alpha \tau} K(0) .
$$


The linearization of the system (1)-(2) about each of the steady-states $(U, W)$ is

$$
\begin{aligned}
& P_{t}+\left[1+\gamma \int_{\mathbb{R}} K(x-y) H(U(y)-\theta d y] P+Q\right. \\
= & \frac{\alpha-\gamma U(x)}{\left|U^{\prime}(0)\right|} K(x) P(0, t)+\frac{\alpha-\gamma U(x)}{\left|U^{\prime}(A)\right|} K(x-A) P(A, t), \\
Q_{t}= & \varepsilon(P-\tau Q),
\end{aligned}
$$

where $(2+2 \tau+\gamma \tau) \theta \neq \alpha \tau$, and $A=A_{*}$ or $A=A^{*}$ is well defined. The eigenvalue problem is

$$
\begin{aligned}
& \lambda \xi+\left[1+\gamma \int_{\mathbb{R}} K(x-y) H(U(y)-\theta)\right] \xi+\eta \\
= & \frac{\alpha-\gamma U(x)}{\left|U^{\prime}(0)\right|} K(x) \xi(0)+\frac{\alpha-\gamma U(x)}{\left|U^{\prime}(A)\right|} K(x-A) \xi(A), \\
\lambda \eta= & \varepsilon(\xi-\tau \eta),
\end{aligned}
$$

equivalently,

$$
\begin{aligned}
& \left(\lambda+1+\gamma \int_{\mathbb{R}} K(x-y) H(U(y)-\theta) d y+\frac{\varepsilon}{\lambda+\tau \varepsilon}\right) \xi \\
= & \frac{\alpha-\gamma U(x)}{\left|U^{\prime}(0)\right|} K(x) \xi(0)+\frac{\alpha-\gamma U(x)}{\left|U^{\prime}(A)\right|} K(x-A) \xi(A), \\
\eta= & \frac{\varepsilon}{\lambda+\tau \varepsilon} \xi .
\end{aligned}
$$

Define $\Omega(\varepsilon)=\{\lambda \in \mathbb{C}: \operatorname{Re} \lambda>-\tau \varepsilon\}$. Define the linear operator

$$
\mathcal{L}(\varepsilon):\left[C^{0}(\mathbb{R}) \bigcap L^{\infty}(\mathbb{R})\right]^{2} \rightarrow\left[C^{0}(\mathbb{R}) \bigcap L^{\infty}(\mathbb{R})\right]^{2}
$$

by

$$
\begin{gathered}
\mathcal{L}(\varepsilon) \psi=-\left(\begin{array}{cc}
1+\gamma\{K *[H(U-\theta)]\} & 1 \\
-\varepsilon & \tau \varepsilon
\end{array}\right) \psi \\
+\left(\begin{array}{ll}
1 & 0 \\
0 & 0
\end{array}\right)\left[\frac{\alpha-\gamma U(x)}{\left|U^{\prime}(0)\right|} K(x) \psi(0)+\frac{\alpha-\gamma U(x)}{\left|U^{\prime}(A)\right|} K(x-A) \psi(A)\right] .
\end{gathered}
$$

The essential spectrum of $\mathcal{L}(\varepsilon)$ consists of all complex numbers satisfying

$$
\operatorname{Re} \lambda=-\frac{1}{2}\left(1+\tau \varepsilon \pm \sqrt{(1-\tau \varepsilon)^{2}-4 \varepsilon}\right) \text {. }
$$

Suppose that $(2+2 \tau+\gamma \tau) \theta \neq \alpha \tau$. Then, as before, the eigenvalues satisfy

$$
\lambda+\frac{\alpha \tau+\gamma \theta}{(\alpha-\gamma \theta) \tau}+\frac{\varepsilon}{\lambda+\tau \varepsilon}=(\alpha-\gamma \theta) \frac{K(0) \pm K(A)}{\left|U^{\prime}(0)\right|} \equiv \Psi_{ \pm},
$$

so

$$
\lambda_{ \pm}=\frac{\Psi_{ \pm}-1-\tau \varepsilon \pm \sqrt{\left(\Psi_{ \pm}-1-\tau \varepsilon\right)^{2}+4\left(\Psi_{ \pm} \gamma-\gamma-1\right) \varepsilon}}{2} .
$$

Suppose that $(2+2 \tau+\gamma \tau) \theta=\alpha \tau$. Then there exists no nonzero eigenvalue of $\mathcal{L}(\varepsilon)$ in the right half plane

$$
\left\{\lambda \in \mathbb{C}: \operatorname{Re} \lambda>-\frac{1}{2}\left(1+\tau \varepsilon-\sqrt{(1-\tau \varepsilon)^{2}-4 \varepsilon}\right)\right\} .
$$


The neutral eigenvalue $\lambda=0$ is simple. Therefore, the steady-states are spectrally stable.

Theorem 5. Let $0<\theta<\frac{\alpha \tau}{1+(1+\gamma) \tau}<\Theta<\frac{(\alpha+\beta) \tau}{1+(1+\gamma+\delta) \tau}$. Then, there exist at least four nontrivial steady-state solutions, which cross the threshold $\theta$ only, to the equations (1)-(2). The steady-states are spectrally unstable relative to (1)-(2), if $(2+2 \tau+\gamma \tau) \theta \neq \alpha \tau$.

Proof. It is very similar to that of Theorem 4 and is omitted.

Theorem 6. Let $0<\theta<\frac{\alpha \tau}{1+(1+\gamma) \tau}<\Theta<\frac{(\alpha+\beta) \tau}{1+(1+\gamma+\delta) \tau}$. Then, there exist at least four nontrivial steady-state solutions, which cross both thresholds $\theta$ and $\Theta$, to the equations (1)-(2). The steady-states are spectrally unstable relative to (1)-(2), if $(2+2 \tau+\gamma \tau) \theta \neq \alpha \tau$.

Proof. It is very similar to that of Theorem 4 and is omitted.

\section{Nonlinear stability and linear instability}

3.1. Nonlinear exponential stability. Consider the existence and exponential stability of constant solutions of the scalar integral-differential equation (8). Obviously, if

$$
0<\theta<\frac{\alpha}{1+\gamma}<\Theta<\frac{\alpha+\beta}{1+\gamma+\delta}
$$

then

$$
U_{0} \equiv 0, U_{1} \equiv \frac{\alpha}{1+\gamma}, U_{2} \equiv \frac{\alpha+\beta}{1+\gamma+\delta},
$$

are the constant solutions. Consider global solutions of the initial value problems $u(x, 0)=u_{0}(x)$ for (8). We pay particular attention to the problems $u_{0}(x) \equiv \theta$ and $u_{0}(x) \equiv \Theta$.

Elementary Lemma. The global solution of the initial value problem

$$
\frac{\partial u}{\partial t}+f(x) u=g(x), u(x, 0)=u_{0}(x),
$$

where $f>0$ on $\mathbb{R}$, is given by

$$
u(x, t)=\exp [-f(x) t] u_{0}(x)+\{1-\exp [-f(x) t]\} \frac{g(x)}{f(x)} .
$$

Proof. It is very easy and omitted.
(I) If $\frac{\alpha+\beta}{1+\gamma+\delta}>\Theta$ and $u_{0}(x) \geq \Theta$ for all $x \in \mathbb{R}$ [or if $\frac{\alpha+\beta}{1+\gamma+\delta} \geq \Theta$ and $u_{0}(x)>\Theta$ for all $x \in \mathbb{R}]$, then a global solution of the initial value problem $u(x, 0)=u_{0}(x)$ is given by

$$
u(x, t)=\frac{\alpha+\beta}{1+\gamma+\delta}+e^{-(1+\gamma+\delta) t}\left[u_{0}(x)-\frac{\alpha+\beta}{1+\gamma+\delta}\right] .
$$

Here $u(x, t)>\Theta$ for all $(x, t) \in \mathbb{R} \times \mathbb{R}^{+}$. Additionally

$$
\lim _{t \rightarrow+\infty} u(x, t)=\frac{\alpha+\beta}{1+\gamma+\delta} .
$$

(II) If $\theta<\frac{\alpha}{1+\gamma}<\Theta$ and $\theta \leq u_{0}(x) \leq \Theta$ for all $x \in \mathbb{R}$ [or if $\theta \leq \frac{\alpha}{1+\gamma} \leq \Theta$ and $\theta<u_{0}(x)<\Theta$ for all $\left.x \in \mathbb{R}\right]$, then a global solution of the initial value problem 
$u(x, 0)=u_{0}(x)$ is given by

$$
u(x, t)=\frac{\alpha}{1+\gamma}+e^{-(1+\gamma) t}\left[u_{0}(x)-\frac{\alpha}{1+\gamma}\right] .
$$

Here $\theta<u(x, t)<\Theta$ for all $(x, t) \in \mathbb{R} \times \mathbb{R}^{+}$. Additionally

$$
\lim _{t \rightarrow+\infty} u(x, t)=\frac{\alpha}{1+\gamma} .
$$

(III) If $u_{0}(x) \leq \theta$ for all $x \in \mathbb{R}$, then a global solution is

$$
u(x, t)=e^{-t} u_{0}(x) .
$$

Note that $u(x, t)<\theta$ for all $(x, t) \in \mathbb{R} \times \mathbb{R}^{+}$. Therefore

$$
\lim _{t \rightarrow+\infty} u(x, t)=0 .
$$

It seems that each of the initial value problems $u(x, 0) \equiv \theta$ and $u(x, 0) \equiv \Theta$ for $(8)$ has at least two stable solutions. Overall, we see that the constant solutions are exponentially stable. Nevertheless, the stability problem becomes very complicated if the initial data cross the threshold $\theta$ or $\Theta$. For each of the above cases, if the initial data is even, then the solution is also even. The initial data can be bounded or unbounded. For examples, $u_{0}(x)=\Theta+\cosh x$ or $u_{0}(x)=1+\Theta+(\tanh x)^{2}$.

To investigate the nonlinear exponential stability of the steady-state solutions of (8), we have to study the initial value problems $u(x, 0)=u_{0}(x)$ for the scalar equation (8), here $u_{0}$ is well defined everywhere but it is not necessarily bounded or continuous. Theorem 7 , Theorem 8 and Theorem 9 are concerned with stability of monotone steady-states.

Theorem 7. Let $(2+\gamma) \theta=\alpha$. (I) Suppose that the initial data satisfy $u_{0}(x) \leq \theta$ for all $x<0$ and $\theta \leq u_{0}(x) \leq \Theta$ for all $x>0$. Then a global solution of the initial value problem for (8) is

$$
\begin{gathered}
u(x, t)=\exp \left\{-\left[1+\gamma \int_{-\infty}^{x} K(\xi) d \xi\right] t\right\} u_{0}(x) \\
+\left\{1-\exp \left[-\left(1+\gamma \int_{-\infty}^{x} K(\xi) d \xi\right) t\right]\right\}\left\{\alpha \int_{-\infty}^{x} K(\xi) d \xi /\left[1+\gamma \int_{-\infty}^{x} K(\xi) d \xi\right]\right\} . \\
u(x, t)<\theta \text { for all } x<0 \text { and } \theta<u(x, t)<\Theta \text { for all } x>0 \text {. Moreover } \\
\lim _{t \rightarrow+\infty} u(x, t)=\alpha \int_{-\infty}^{x} K(\xi) d \xi /\left[1+\gamma \int_{-\infty}^{x} K(\xi) d \xi\right] .
\end{gathered}
$$

(II) Suppose that the initial data satisfy $\theta \leq u_{0}(x) \leq \Theta$ for all $x<0$ and $u_{0}(x) \leq \theta$ for all $x>0$. Then a global solution of the initial value problem for (8) is

$$
\begin{gathered}
u(x, t)=\exp \left\{-\left[1+\gamma \int_{x}^{\infty} K(\xi) d \xi\right] t\right\} u_{0}(x) \\
+\left\{1-\exp \left[-\left(1+\gamma \int_{x}^{\infty} K(\xi) d \xi\right) t\right]\right\}\left\{\alpha \int_{x}^{\infty} K(\xi) d \xi /\left[1+\gamma \int_{x}^{\infty} K(\xi) d \xi\right]\right\} . \\
\theta<u(x, t)<\Theta \text { for all } x<0 \text { and } u(x, t)<\theta \text { for all } x>0 \text {. Moreover } \\
\lim _{t \rightarrow+\infty} u(x, t)=\alpha \int_{x}^{\infty} K(\xi) d \xi /\left[1+\gamma \int_{x}^{\infty} K(\xi) d \xi\right] .
\end{gathered}
$$


Proof. (I) We are looking for solutions satisfying the conditions $u(x, t)<\theta$ for $x<0$ and $\theta<u(x, t)<\Theta$ for $x>0$. Therefore, the integral-differential equation (8) reduces to the equation

$$
\frac{\partial u}{\partial t}+u=(\alpha-\gamma u) \int_{-\infty}^{x} K(\xi) d \xi
$$

Formally, we can treat it as an ordinary differential equation in terms of $t$ with $x$ being a real parameter. The integrating factor is

$$
\exp \left\{\left[1+\gamma \int_{-\infty}^{x} K(\xi) d \xi\right] t\right\}
$$

Now it is straightforward to derive the solution formula. If $x<0$, then $u_{0}(x) \leq \theta$ and

$$
\begin{gathered}
\alpha \int_{-\infty}^{x} K(\xi) d \xi /\left[1+\gamma \int_{-\infty}^{x} K(\xi) d \xi\right] \\
<\alpha \int_{-\infty}^{0} K(\xi) d \xi /\left[1+\gamma \int_{-\infty}^{0} K(\xi) d \xi\right]=\frac{\alpha}{2+\gamma}=\theta .
\end{gathered}
$$

Thus $u(x, t)<\theta$. Similarly, if $x>0$, then $\theta \leq u_{0}(x) \leq \Theta$ and

$$
\begin{gathered}
\alpha \int_{-\infty}^{x} K(\xi) d \xi /\left[1+\gamma \int_{-\infty}^{x} K(\xi) d \xi\right] \\
<\alpha \int_{-\infty}^{\infty} K(\xi) d \xi /\left[1+\gamma \int_{-\infty}^{\infty} K(\xi) d \xi\right]=\frac{\alpha}{1+\gamma}<\Theta
\end{gathered}
$$

and

$$
\begin{gathered}
\alpha \int_{-\infty}^{x} K(\xi) d \xi /\left[1+\gamma \int_{-\infty}^{x} K(\xi) d \xi\right] \\
>\alpha \int_{-\infty}^{0} K(\xi) d \xi /\left[1+\gamma \int_{-\infty}^{0} K(\xi) d \xi\right]=\frac{\alpha}{2+\gamma}=\theta .
\end{gathered}
$$

Thus $\theta<u(x, t)<\Theta$. Other details are omitted. (II) is proved similarly. \#

Theorem 8. Let $(2+2 \gamma+\delta) \Theta=2 \alpha+\beta$. (I) Suppose that the initial data satisfy $\theta \leq u_{0}(x) \leq \Theta$ for all $x<0$ and $u_{0}(x) \geq \Theta$ for all $x>0$. Then a global solution of the initial value problem for (8) is

$$
\begin{aligned}
u(x, t)= & \exp \left\{-\left[1+\gamma+\delta \int_{-\infty}^{x} K(\xi) d \xi\right] t\right\} u_{0}(x) \\
& +\left\{1-\exp \left[-\left(1+\gamma+\delta \int_{-\infty}^{x} K(\xi) d \xi\right) t\right]\right\} \\
& \left\{\left[\alpha+\beta \int_{-\infty}^{x} K(\xi) d \xi\right] /\left[1+\gamma+\delta \int_{-\infty}^{x} K(\xi) d \xi\right]\right\} .
\end{aligned}
$$

$\theta<u(x, t)<\Theta$ for all $x<0$ and $u(x, t)>\Theta$ for all $x>0$. Moreover

$$
\lim _{t \rightarrow+\infty} u(x, t)=\left[\alpha+\beta \int_{-\infty}^{x} K(\xi) d \xi\right] /\left[1+\gamma+\delta \int_{-\infty}^{x} K(\xi) d \xi\right] .
$$


(II) Suppose that the initial data satisfy $u_{0}(x) \geq \Theta$ for all $x<0$ and $\theta \leq u_{0}(x) \leq \Theta$ for all $x>0$. Then a global solution of the initial value problem for (8) is

$$
\begin{aligned}
u(x, t)= & \exp \left\{-\left[1+\gamma+\delta \int_{x}^{\infty} K(\xi) d \xi\right] t\right\} u_{0}(x) \\
& +\left\{1-\exp \left[-\left(1+\gamma+\delta \int_{x}^{\infty} K(\xi) d \xi\right) t\right]\right\} \\
& \left\{\left[\alpha+\beta \int_{x}^{\infty} K(\xi) d \xi\right] /\left[1+\gamma+\delta \int_{x}^{\infty} K(\xi) d \xi\right]\right\} .
\end{aligned}
$$

$u(x, t)>\Theta$ for all $x<0$ and $\theta<(x, t)<\Theta$ for all $x>0$. Moreover

$$
\lim _{t \rightarrow+\infty} u(x, t)=\left[\alpha+\beta \int_{x}^{\infty} K(\xi) d \xi\right] /\left[1+\gamma+\delta \int_{x}^{\infty} K(\xi) d \xi\right] .
$$

Proof. It is very similar to that of Theorem 7 and is omitted.

Theorem 9. Suppose that $(\alpha-\gamma \Theta)[(2+\delta) \Theta-\beta]>0$ and $(\beta-\delta \theta)[(2+\gamma) \theta-\alpha]>$ 0 , such that

$$
\frac{(2+\gamma) \theta-\alpha}{2(\beta-\delta \theta)}+\frac{(2+\delta) \Theta-\beta}{2(\alpha-\gamma \Theta)}=1
$$

(I) Suppose that the initial data satisfy $u_{0}(x) \leq \theta$ for all $x<0$ and $u_{0}(x) \geq \theta$ for all $x>0 ; u_{0}(x) \leq \Theta$ for all $x<\Omega_{*}$ and $u_{0}(x) \geq \Theta$ for all $x>\Omega_{*}$. Then a global solution of the initial value problem for (8) is

$$
\begin{gathered}
u(x, t)=\exp \left\{-\left[1+\gamma \int_{-\infty}^{x} K(\xi) d \xi+\delta \int_{-\infty}^{x-\Omega_{*}} K(\xi) d \xi\right] t\right\} u_{0}(x) \\
+\left\{1-\exp \left[-\left(1+\gamma \int_{-\infty}^{x} K(\xi) d \xi+\delta \int_{-\infty}^{x-\Omega_{*}} K(\xi) d \xi\right) t\right]\right\} \\
\times\left\{\alpha \int_{-\infty}^{x} K(\xi) d \xi+\beta \int_{-\infty}^{x-\Omega_{*}} K(\xi) d \xi /\left[1+\gamma \int_{-\infty}^{x} K(\xi) d \xi+\delta \int_{-\infty}^{x-\Omega_{*}} K(\xi) d \xi\right]\right\} .
\end{gathered}
$$

$u(x, t)<\theta$ for all $x<0$ and $u(x, t)>\theta$ for all $x>0 ; u(x, t)<\Theta$ for all $x<\Omega_{*}$ and $u(x, t)>\Theta$ for all $x>\Omega_{*}$. Moreover

$$
\begin{aligned}
\lim _{t \rightarrow+\infty} u(x, t)= & {\left[\alpha \int_{-\infty}^{x} K(\xi) d \xi+\beta \int_{-\infty}^{x-\Omega_{*}} K(\xi) d \xi\right] } \\
& /\left[1+\gamma \int_{-\infty}^{x} K(\xi) d \xi+\delta \int_{-\infty}^{x-\Omega_{*}} K(\xi) d \xi\right] .
\end{aligned}
$$

(II) Suppose that the initial data satisfy $u_{0}(x) \geq \theta$ for all $x<0$ and $u_{0}(x) \leq \theta$ for all $x>0 ; u_{0}(x) \geq \Theta$ for all $x<\Omega^{*}$ and $u_{0}(x) \leq \Theta$ for all $x>\Omega^{*}$. Then a global solution of the initial value problem for (8) is

$$
\begin{aligned}
& u(x, t)=\exp \left\{-\left[1+\gamma \int_{x}^{\infty} K(\xi) d \xi+\delta \int_{x-\Omega^{*}}^{\infty} K(\xi) d \xi\right] t\right\} u_{0}(x) \\
& +\left\{1-\exp \left[-\left(1+\gamma \int_{x}^{\infty} K(\xi) d \xi+\delta \int_{x-\Omega^{*}}^{\infty} K(\xi) d \xi\right) t\right]\right\}
\end{aligned}
$$


$\times\left\{\left[\alpha \int_{x}^{\infty} K(\xi) d \xi+\beta \int_{x-\Omega^{*}}^{\infty} K(\xi) d \xi\right] /\left[1+\gamma \int_{x}^{\infty} K(\xi) d \xi+\delta \int_{x-\Omega^{*}}^{\infty} K(\xi) d \xi\right]\right\}$. $u(x, t)>\theta$ for all $x<0$ and $u(x, t)<\theta$ for all $x>0 ; u(x, t)>\Theta$ for all $x<\Omega^{*}$ and $u(x, t)<\Theta$ for all $x>\Omega^{*}$. Moreover

$$
\begin{aligned}
\lim _{t \rightarrow+\infty} u(x, t)= & {\left[\alpha \int_{x}^{\infty} K(\xi) d \xi+\beta \int_{x-\Omega^{*}}^{\infty} K(\xi) d \xi\right] } \\
& /\left[1+\gamma \int_{x}^{\infty} K(\xi) d \xi+\delta \int_{x-\Omega^{*}}^{\infty} K(\xi) d \xi\right] .
\end{aligned}
$$

Proof. It is very similar to that of Theorem 7 and is omitted.

Theorem 10, Theorem 11 and Theorem 12 are concerned with stability of steady-states with a unique maximum/minimum at some point. Recall that $X_{*}, Y_{*}$, $Z_{*}$ or $X^{*}, Y^{*}, Z^{*}$ are the numbers found in Theorem 1, Theorem 2, and Theorem 3 , respectively.

Theorem 10. (I) Let $(2+\gamma) \theta<\alpha$. Suppose that the initial data satisfy $u_{0}(x) \leq \theta$ for all $x<0$ and $x>X_{*}$, and $\theta \leq u_{0}(x) \leq \Theta$ for all $0<x<X_{*}$. Then a global solution of the initial value problem for (8) is

$$
\begin{aligned}
u(x, t)= & \exp \left\{-\left[1+\gamma \int_{x-X_{*}}^{x} K(\xi) d \xi\right] t\right\} u_{0}(x) \\
& +\left\{1-\exp \left[-\left(1+\gamma \int_{x-X_{*}}^{x} K(\xi) d \xi\right) t\right]\right\} \\
& \left\{\alpha \int_{x-X_{*}}^{x} K(\xi) d \xi /\left[1+\gamma \int_{x-X_{*}}^{x} K(\xi) d \xi\right]\right\} .
\end{aligned}
$$

$u(x, t)<\theta$ for $x<0$ and $x>X_{*}$, and $\theta<u(x, t)<\Theta$ for all $0<x<X_{*}$. Moreover

$$
\lim _{t \rightarrow+\infty} u(x, t)=\alpha \int_{x-X_{*}}^{x} K(\xi) d \xi /\left[1+\gamma \int_{x-X_{*}}^{x} K(\xi) d \xi\right] .
$$

(II) Let $(2+\gamma) \theta>\alpha$. Suppose that the initial data satisfy $\theta \leq u_{0}(x) \leq \Theta$ for all $x<0$ and $x>X^{*}$, and $u_{0}(x) \leq \theta$ for all $0<x<X^{*}$. Then a global solution of the initial value problem for (8) is

$$
\begin{aligned}
u(x, t)= & \exp \left\{-\left[1+\gamma-\gamma \int_{x-X^{*}}^{x} K(\xi) d \xi\right] t\right\} u_{0}(x) \\
& +\left\{1-\exp \left[-\left(1+\gamma-\gamma \int_{x-X^{*}}^{x} K(\xi) d \xi\right) t\right]\right\} \\
& \left\{\left[\alpha-\alpha \int_{x-X^{*}}^{x} K(\xi) d \xi\right] /\left[1+\gamma-\gamma \int_{x-X^{*}}^{x} K(\xi) d \xi\right]\right\} .
\end{aligned}
$$

$\theta<u(x, t)<\Theta$ for all $x<0$ and $x>X^{*}$ and $u(x, t)<\theta$ for all $0<x<X^{*}$. Moreover

$$
\lim _{t \rightarrow+\infty} u(x, t)=\left[\alpha-\alpha \int_{x-X^{*}}^{x} K(\xi) d \xi\right] /\left[1+\gamma-\gamma \int_{x-X^{*}}^{x} K(\xi) d \xi\right] .
$$

Proof. (I) We are looking for solutions satisfying the conditions $u(x, t)<\theta$ for $x<0$ and $x>X_{*}$ and $\theta<u(x, t)<\Theta$ for $0<x<X_{*}$. The integral-differential 
equation (8) reduces to the equation

$$
\frac{\partial u}{\partial t}+u=(\alpha-\gamma u) \int_{x-X_{*}}^{x} K(\xi) d \xi
$$

Formally, we can treat it as an ordinary differential equation in terms of $t$ with $x$ being a real parameter. The integrating factor is

$$
\exp \left\{\left[1+\gamma \int_{x-X_{*}}^{x} K(\xi) d \xi\right] t\right\} .
$$

Other details are omitted. (II) is proved similarly.

Theorem 11. (I) Let $(2+2 \gamma+\delta) \Theta<2 \alpha+\beta$. Suppose that the initial data satisfy $\theta \leq u_{0}(x) \leq \Theta$ for all $x<0$ and $x>Y_{*}$, and $u_{0}(x) \geq \Theta$ for all $0<x<Y_{*}$. Then a global solution of the initial value problem for (8) is

$$
\begin{aligned}
u(x, t)= & \exp \left\{-\left[1+\gamma+\delta \int_{x-Y_{*}}^{x} K(\xi) d \xi\right] t\right\} u_{0}(x) \\
& +\left\{1-\exp \left[-\left(1+\gamma+\delta \int_{x-Y_{*}}^{x} K(\xi) d \xi\right) t\right]\right\} \\
& \left\{\left[\alpha+\beta \int_{x-Y_{*}}^{x} K(\xi) d \xi\right] /\left[1+\gamma+\delta \int_{x-Y_{*}}^{x} K(\xi) d \xi\right]\right\}
\end{aligned}
$$

$\theta<u(x, t)<\Theta$ for all $x<0$ and $x>Y_{*}$, and $u(x, t)>\Theta$ for all $0<x<Y_{*}$. Moreover

$$
\lim _{t \rightarrow+\infty} u(x, t)=\left[\alpha+\beta \int_{x-Y_{*}}^{x} K(\xi) d \xi\right] /\left[1+\gamma+\delta \int_{x-Y_{*}}^{x} K(\xi) d \xi\right] .
$$

(II) Let $(2+2 \gamma+\delta) \Theta>2 \alpha+\beta$. Suppose that the initial data satisfy $u_{0}(x) \geq \Theta$ for all $x<0$ and $x>Y^{*}$, and $\theta \leq u_{0}(x) \leq \Theta$ for all $0<x<Y^{*}$. Then a global solution of the initial value problem for (8) is

$$
\begin{aligned}
u(x, t)= & \exp \left\{-\left[1+\gamma+\delta-\delta \int_{x-Y^{*}}^{x} K(\xi) d \xi\right] t\right\} u_{0}(x) \\
& +\left\{1-\exp \left[-\left(1+\gamma+\delta-\delta \int_{x-Y^{*}}^{x} K(\xi) d \xi\right) t\right]\right\} \\
& \left\{\left[\alpha+\beta-\beta \int_{x-Y^{*}}^{x} K(\xi) d \xi\right] /\right. \\
& {\left.\left[1+\gamma+\delta-\delta \int_{x-Y^{*}}^{x} K(\xi) d \xi\right]\right\} . }
\end{aligned}
$$

$u(x, t)>\Theta$ for all $x<0$ and $x>Y^{*}$, and $\theta<u(x, t)<\Theta$ for all $0<x<Y^{*}$. Moreover

$$
\lim _{t \rightarrow+\infty} u(x, t)=\left[\alpha+\beta-\beta \int_{x-Y^{*}}^{x} K(\xi) d \xi\right] /\left[1+\gamma+\delta-\delta \int_{x-Y^{*}}^{x} K(\xi) d \xi\right] .
$$

Proof. It is very similar to that of Theorem 10 and is omitted.

Theorem 12. Suppose that $(\alpha-\gamma \Theta)[(2+\delta) \Theta-\beta]>0$ and $(\beta-\delta \theta)[(2+\gamma) \theta-$ $\alpha]>0$, such that

$$
\frac{(2+\gamma) \theta-\alpha}{2(\beta-\delta \theta)}+\frac{(2+\delta) \Theta-\beta}{2(\alpha-\gamma \Theta)} \neq 1 .
$$


(I) Suppose that the initial data satisfy $u_{0}(x) \leq \theta$ for all $x<0$ and $x>Z_{*}$, and $u_{0}(x) \geq \theta$ for all $0<x<Z_{*} ; u_{0}(x) \leq \Theta$ for all $x<\Gamma_{*}$ and $x>\Lambda_{*}$, and $u_{0}(x) \geq \Theta$ for all $\Gamma_{*}<x<\Lambda_{*}$ Then a global solution of the initial value problem for (8) is

$$
\begin{aligned}
u(x, t)= & \exp \left\{-\left[1+\gamma \int_{x-Z_{*}}^{x} K(\xi) d \xi+\delta \int_{x-\Lambda_{*}}^{x-\Gamma_{*}} K(\xi) d \xi\right] t\right\} u_{0}(x) \\
& +\left\{1-\exp \left[-\left(1+\gamma \int_{x-Z_{*}}^{x} K(\xi) d \xi+\delta \int_{x-\Lambda_{*}}^{x-\Gamma_{*}} K(\xi) d \xi\right) t\right]\right\} \\
& \times\left\{\left[\alpha \int_{x-Z_{*}}^{x} K(\xi) d \xi+\beta \int_{x-\Lambda_{*}}^{x-\Gamma_{*}} K(\xi) d \xi\right]\right. \\
& \left./\left[1+\gamma \int_{x-Z_{*}}^{x} K(\xi) d \xi+\delta \int_{x-\Lambda_{*}}^{x-\Gamma_{*}} K(\xi) d \xi\right]\right\} .
\end{aligned}
$$

$u(x, t)<\theta$ for all $x<0$ and $x>Z_{*}$, and $u(x, t)>\theta$ for all $0<x<Z_{*} ; u(x, t)<\Theta$ for all $x<\Gamma_{*}$ and $x>\Lambda_{*}$, and $u(x, t)>\Theta$ for all $\Gamma_{*}<x<\Lambda_{*}$ Moreover

$$
\begin{aligned}
\lim _{t \rightarrow+\infty} u(x, t)= & {\left[\alpha \int_{x-Z_{*}}^{x} K(\xi) d \xi+\beta \int_{x-\Lambda_{*}}^{x-\Gamma_{*}} K(\xi) d \xi\right] } \\
& /\left[1+\gamma \int_{x-Z_{*}}^{x} K(\xi) d \xi+\delta \int_{x-\Lambda_{*}}^{x-\Gamma_{*}} K(\xi) d \xi\right] .
\end{aligned}
$$

(II) Suppose that the initial data satisfy $u_{0}(x) \geq \theta$ for all $x<0$ and $x>Z^{*}$, and $u_{0}(x) \leq \theta$ for all $0<x<Z^{*} ; u_{0}(x) \geq \Theta$ for all $x<\Gamma^{*}$ and $x>\Lambda^{*}$, and $u_{0}(x) \leq \Theta$ for all $\Gamma^{*}<x<\Lambda^{*}$. Then a global solution of the initial value problem for (8) is

$$
\begin{aligned}
u(x, t)= & \exp \left\{-\left[1+\gamma-\gamma \int_{x-Z^{*}}^{x} K(\xi) d \xi+\delta-\delta \int_{x-\Lambda^{*}}^{x-\Gamma^{*}} K(\xi) d \xi\right] t\right\} u_{0}(x) \\
& +\left\{1-\exp \left[-\left(1+\gamma-\gamma \int_{x-Z^{*}}^{x} K(\xi) d \xi+\delta-\delta \int_{x-\Lambda^{*}}^{x-\Gamma^{*}} K(\xi) d \xi\right) t\right]\right\} \\
& \times\left\{\left[\alpha-\alpha \int_{x-Z^{*}}^{x} K(\xi) d \xi+\beta-\beta \int_{x-\Lambda^{*}}^{x-\Gamma^{*}} K(\xi) d \xi\right]\right. \\
& \left./\left[1+\gamma-\gamma \int_{x-Z^{*}}^{x} K(\xi) d \xi+\delta-\delta \int_{x-\Lambda^{*}}^{x-\Gamma^{*}} K(\xi) d \xi\right]\right\} .
\end{aligned}
$$

$u(x, t)>\theta$ for all $x<0$ and $x>Z^{*}$ and $u(x, t)<\theta$ for all $0<x<Z^{*} ; u(x, t)>\Theta$ for all $x<\Gamma^{*}$ and $x>\Lambda^{*}$, and $u(x, t)<\Theta$ for all $\Gamma^{*}<x<\Lambda^{*}$ Moreover

$$
\begin{aligned}
\lim _{t \rightarrow+\infty} u(x, t)= & {\left[\alpha-\alpha \int_{x-Z^{*}}^{x} K(\xi) d \xi+\beta \int_{x-\Lambda^{*}}^{x-\Gamma^{*}} K(\xi) d \xi\right] } \\
& /\left[1+\gamma-\gamma \int_{x-Z^{*}}^{x} K(\xi) d \xi+\delta-\delta \int_{x-\Lambda^{*}}^{x-\Gamma^{*}} K(\xi) d \xi\right] .
\end{aligned}
$$

Proof. It is very similar to that of Theorem 10 and is omitted. 
3.2. Linear exponential instability. We first discuss the cases $(2+\gamma) \theta \neq \alpha, \quad$ or $\quad(2+2 \gamma+\delta) \Theta \neq 2 \alpha+\beta, \quad$ or $\quad \frac{(2+\gamma) \theta-\alpha}{2(\beta-\delta \theta)}+\frac{(2+\delta) \Theta-\beta}{2(\alpha-\gamma \Theta)} \neq 1$ so that $X_{*}, Y_{*}, Z_{*}, X^{*}, Y^{*}, Z^{*}$ are well defined. We then consider the cases $(2+\gamma) \theta=\alpha$, or $(2+2 \gamma+\delta) \Theta=2 \alpha+\beta, \quad$ or $\quad \frac{(2+\gamma) \theta-\alpha}{2(\beta-\delta \theta)}+\frac{(2+\delta) \Theta-\beta}{2(\alpha-\gamma \Theta)}=1$.

Theorem 13. (I) Let $(1+\gamma) \theta<\alpha$ and $(2+\gamma) \theta \neq \alpha$. Then, the steady-states are linearly exponentially unstable relative to the equation

$$
\begin{gathered}
P_{t}+\left[1+\gamma \int_{\mathbb{R}} K(x-y) H(U(y)-\theta) d y\right] P \\
=[\alpha-\gamma U(x)]\left[\frac{K(x)}{\left|U^{\prime}(0)\right|} P(0, t)+\frac{K(x-X)}{\left|U^{\prime}(X)\right|} P(X, t)\right],
\end{gathered}
$$

where $X=X_{*}$ or $X=X^{*}$. (II) Let $(1+\gamma) \theta<\alpha$ and $(2+\gamma) \theta=\alpha$. Then, the steady-states are linearly exponentially stable relative to the equation

$$
\begin{gathered}
P_{t}+\left[1+\gamma \int_{\mathbb{R}} K(x-y) H(U(y)-\theta) d y\right] P \\
=[\alpha-\gamma U(x)] \frac{K(x)}{\left|U^{\prime}(0)\right|} P(0, t) .
\end{gathered}
$$

Proof. (I) It is not difficult to solve the linear equation to obtain the global solution

$$
\begin{aligned}
P(x, t) & =\exp \left\{-t\left[1+\gamma \int_{\mathbb{R}} K(x-y) H(U(y)-\theta) d y\right]\right\} P_{0}(x) \\
& +\int_{0}^{t} \exp \left\{(s-t)\left[1+\gamma \int_{\mathbb{R}} K(x-y) H(U(y)-\theta) d y\right]\right\} \\
& \times[\alpha-\gamma U(x)]\left[\frac{K(x)}{\left|U^{\prime}(0)\right|} P(0, s)+\frac{K(x-X)}{\left|U^{\prime}(X)\right|} P(X, s)\right] d s .
\end{aligned}
$$

To find $P(0, t)$ and $P(X, t)$, by setting $x=0$ and $x=X$, respectively, we obtain the equations

$$
\begin{aligned}
P_{t}(0, t) & =\left[(\alpha-\gamma \theta) \frac{K(0)}{\left|U^{\prime}(0)\right|}-\frac{\alpha}{\alpha-\gamma \theta}\right] P(0, t)+(\alpha-\gamma \theta) \frac{K(X)}{\left|U^{\prime}(X)\right|} P(X, t), \\
P_{t}(X, t) & =\left[(\alpha-\gamma \theta) \frac{K(0)}{\left|U^{\prime}(X)\right|}-\frac{\alpha}{\alpha-\gamma \theta}\right] P(X, t)+(\alpha-\gamma \theta) \frac{K(X)}{\left|U^{\prime}(0)\right|} P(0, t) .
\end{aligned}
$$

The eigenvalues and eigenvectors of the coefficient matrix

$$
\left(\begin{array}{cc}
(\alpha-\gamma \theta) \frac{K(0)}{\left|U^{\prime}(0)\right|}-\frac{\alpha}{\alpha-\gamma \theta} & (\alpha-\gamma \theta) \frac{K(X)}{\left|U^{\prime}(X)\right|} \\
(\alpha-\gamma \theta) \frac{K(X)}{\left|U^{\prime}(0)\right|} & (\alpha-\gamma \theta) \frac{K(0)}{\left|U^{\prime}(X)\right|}-\frac{\alpha}{\alpha-\gamma \theta}
\end{array}\right)=(\alpha-\gamma \theta) \frac{K(X)}{\left|U^{\prime}(0)\right|}\left(\begin{array}{ll}
1 & 1 \\
1 & 1
\end{array}\right)
$$

are

$\lambda_{1}=(\alpha-\gamma \theta) \frac{K(0)-K(X)}{\left|U^{\prime}(0)\right|}-\frac{\alpha}{\alpha-\gamma \theta}=0, \lambda_{2}=(\alpha-\gamma \theta) \frac{K(0)+K(X)}{\left|U^{\prime}(0)\right|}-\frac{\alpha}{\alpha-\gamma \theta}>0$, and

$$
\left(\begin{array}{l}
\xi_{1} \\
\eta_{1}
\end{array}\right)=\left(\begin{array}{c}
1 \\
-1
\end{array}\right),\left(\begin{array}{l}
\xi_{2} \\
\eta_{2}
\end{array}\right)=\left(\begin{array}{l}
1 \\
1
\end{array}\right)
$$


respectively. Therefore, we can obtain

$$
\left(\begin{array}{c}
P(0, t) \\
P(X, t)
\end{array}\right)=C_{1}\left(\begin{array}{c}
1 \\
-1
\end{array}\right)+C_{2}\left(\begin{array}{l}
1 \\
1
\end{array}\right) \exp \left(\lambda_{2} t\right)
$$

To find the constants $C_{1}$ and $C_{2}$, we use the initial data $P(x, 0)=P_{0}(x)$. It is straightforward to find that

$$
C_{1}=\frac{P(0,0)-P(X, 0)}{2}, C_{2}=\frac{P(0,0)+P(X, 0)}{2} .
$$

Therefore

$$
\begin{aligned}
P(0, t) & =\frac{P(0,0)-P(X, 0)}{2}+\frac{P(0,0)+P(X, 0)}{2} \exp \left(\lambda_{2} t\right), \\
P(X, t) & =\frac{P(X, 0)-P(0,0)}{2}+\frac{P(0,0)+P(X, 0)}{2} \exp \left(\lambda_{2} t\right) .
\end{aligned}
$$

Now the global solution of the initial value problem $P(x, 0)=P_{0}(x)$ for the linear equation is given by

$$
\begin{aligned}
P(x, t) & =\exp \left\{-t\left[1+\gamma \int_{\mathbb{R}} K(x-y) H(U(y)-\theta) d y\right]\right\} P_{0}(x) \\
& +\int_{0}^{t} \exp \left\{(s-t)\left[1+\gamma \int_{\mathbb{R}} K(x-y) H(U(y)-\theta) d y\right]\right\} \\
& \times[\alpha-\gamma U(x)]\left[\frac{K(x)}{\left|U^{\prime}(0)\right|} P(0, s)+\frac{K(x-X)}{\left|U^{\prime}(X)\right|} P(X, s)\right] d s .
\end{aligned}
$$

Therefore

$$
\lim _{t \rightarrow+\infty}|P(x, t)|=+\infty .
$$

This proves the instability of the steady-states. The case $(2+\gamma) \theta=\alpha$ is proved similarly.

Theorem 14. (I) Let $(2+2 \gamma+\delta) \Theta \neq 2 \alpha+\beta$. Then, the steady-states are linearly exponentially unstable relative to the equation

$$
\begin{aligned}
& P_{t}+\left[1+\gamma+\delta \int_{\mathbb{R}} K(x-y) H(U(y)-\Theta) d y\right] P \\
= & {[\beta-\delta U(x)]\left[\frac{K(x)}{\left|U^{\prime}(0)\right|} P(0, t)+\frac{K(x-Y)}{\left|U^{\prime}(Y)\right|} P(Y, t)\right], }
\end{aligned}
$$

where $Y=Y_{*}$ or $Y=Y^{*}$. (II) Let $(2+2 \gamma+\delta) \Theta=2 \alpha+\beta$. Then, the steady-states are linearly exponentially stable relative to the equation

$$
\begin{gathered}
P_{t}+\left[1+\gamma+\delta \int_{\mathbb{R}} K(x-y) H(U(y)-\Theta) d y\right] P \\
=[\beta-\delta U(x)] \frac{K(x)}{\left|U^{\prime}(0)\right|} P(0, t) .
\end{gathered}
$$

Proof. It is very similar to that of Theorem 13 and is omitted.

Theorem 15. (I) Suppose that $(\alpha-\gamma \Theta)[(2+\delta) \Theta-\beta]>0$ and $(\beta-\delta \theta)[(2+$ $\gamma) \theta-\alpha]>0$, such that

$$
\frac{(2+\gamma) \theta-\alpha}{2(\beta-\delta \theta)}+\frac{(2+\delta) \Theta-\beta}{2(\alpha-\gamma \Theta)} \neq 1
$$


Then, the steady-states are linearly exponentially unstable relative to the equation

$$
\begin{aligned}
P_{t}+[1+\gamma & \left.\int_{\mathbb{R}} K(x-y) H(U(y)-\theta) d y+\delta \int_{\mathbb{R}} K(x-y) H(U(y)-\Theta) d y\right] P \\
= & {[\alpha-\gamma U(x)]\left[\frac{K(x)}{\left|U^{\prime}(0)\right|} P(0, t)+\frac{K(x-Z)}{\left|U^{\prime}(Z)\right|} P(Z, t)\right] } \\
+ & {[\beta-\delta U(x)]\left[\frac{K(x-\Gamma)}{\left|U^{\prime}(\Gamma)\right|} P(\Gamma, t)+\frac{K(x-\Lambda)}{\left|U^{\prime}(\Lambda)\right|} P(\Lambda, t)\right], }
\end{aligned}
$$

where $Z=Z_{*}, \Gamma=\Gamma_{*}, \Lambda=\Lambda_{*}$; or $Z=Z^{*}, \Gamma=\Gamma^{*}, \Lambda=\Lambda^{*}$. (II) Suppose that $(\alpha-\gamma \Theta)[(2+\delta) \Theta-\beta]>0$ and $(\beta-\delta \theta)[(2+\gamma) \theta-\alpha]>0$, such that

$$
\frac{(2+\gamma) \theta-\alpha}{2(\beta-\delta \theta)}+\frac{(2+\delta) \Theta-\beta}{2(\alpha-\gamma \Theta)}=1
$$

Then, the steady-states are linearly exponentially stable relative to the equation

$$
\begin{aligned}
P_{t}+\left[1+\gamma \int_{\mathbb{R}} K(x-y)\right. & \left.H(U(y)-\theta) d y+\delta \int_{\mathbb{R}} K(x-y) H(U(y)-\Theta) d y\right] P \\
= & {[\alpha-\gamma U(x)] \frac{K(x)}{\left|U^{\prime}(0)\right|} P(0, t) } \\
+ & {[\beta-\delta U(x)] \frac{K(x-\Omega)}{\left|U^{\prime}(\Omega)\right|} P(\Omega, t), }
\end{aligned}
$$

where $\Omega=\Omega_{*}$ or $\Omega=\Omega^{*}$.

Proof. It is very similar to that of Theorem 13 and is omitted.

Remark . The initial value problems $(u(x, 0), w(x, 0))=\left(u_{0}(x), w_{0}(x)\right)$ for the system (1)-(2) can be studied similarly.

Remark . Similar results may be extended to the $n$-dimensional nonlocal equations

$$
\begin{aligned}
\frac{\partial u}{\partial t}+u+w & =(\alpha-\gamma u) \int_{\mathbb{R}^{n}} K(x-y) H(u(y, t)-\theta) d y \\
& +(\beta-\delta u) \int_{\mathbb{R}^{n}} K(x-y) H(u(y, t)-\Theta) d y \\
\frac{\partial w}{\partial t} & =\varepsilon(u-\tau w),
\end{aligned}
$$

where $n \geq 2$ is an integer.

Remark . The results in this paper strongly indicate that the linearized stability criterion is not valid at least for steady-state solutions of nonlocal equations involving the Heaviside step function.

\section{Bifurcations}

To simplify our analysis, we only consider initial data identically equal to constants. It was proved in Section 3 that the initial value problems for (8) with initial data $u_{0} \equiv \theta \pm \kappa$ or $u_{0} \equiv \Theta \pm \kappa$, where $0<\kappa \ll 1$, have a unique global solution, respectively. Nevertheless, if $\kappa=0$, then there are at least four global solutions corresponding to the initial data $u_{0} \equiv \theta$ or $u_{0} \equiv \Theta$, respectively. Therefore, $u_{0} \equiv \theta$ and $u_{0} \equiv \Theta$ are bifurcation points. 


\section{Appendix}

We briefly discuss steady-states of the following nonlinear singularly perturbed systems of integral-differential equations

$$
\begin{aligned}
\frac{\partial u}{\partial t}+\phi(u)+w & =(\alpha-\gamma u) \int_{\mathbb{R}} K(x-y) H(u(y, t)-\theta) d y, \\
\frac{\partial w}{\partial t}+\varepsilon w & =\varepsilon g(u), \quad \text { in } \mathbb{R} \times \mathbb{R}^{+} .
\end{aligned}
$$

Theorem 16. Let $\phi(\theta)<\alpha-\gamma \theta$. Suppose that the kernel is nonnegative everywhere. Then, there exists at least four steady-state solutions $U=U(\cdot)$ to (5). Suppose that the kernel is a Mexican hat function such that $|K(x)| \leq C \exp (-\rho|x|)$ on $\mathbb{R}$ hold, for some positive constants $C$ and $\rho$. Let $\phi^{\prime}>0$ and $\gamma=0$. Then, there is at least a steady-state. In both cases, the steady-states are unstable, in the sense of $L^{\infty}(\mathbb{R})$-norm.

The steady-state solution serves as a slow singular homoclinic orbit for (5)-(6). If we perturb $\varepsilon>0$, then we get a slow pulse as well as a fast pulse.

There are two stable constant steady-states $U \equiv 0$ and $U \equiv \beta$ to (5). These stable steady-states correspond to neurons in their resting states. Let us search for non-constant steady-states.

Proof of Theorem 16. (A) First of all, let the kernel be a nonnegative function. The steady-state solutions $U=U(\cdot)$ satisfy

$$
\phi(U)=(\alpha-\gamma U) \int_{\mathbb{R}} K(x-y) H(U(y)-\theta) d y .
$$

(I) If $U<\theta$ on $(-\infty, 0) \bigcup\left(X_{*},+\infty\right)$ and $U>\theta$ on $\left(0, X_{*}\right)$, for some number $X_{*}>0$, then

$$
\int_{\mathbb{R}} K(x-y) H(U(y)-\theta) d y=\int_{x-X_{*}}^{x} K(\xi) d \xi \equiv g_{1}(x) .
$$

(II) If $U>\theta$ on $(-\infty, 0) \bigcup\left(X^{*},+\infty\right)$ and $U<\theta$ on $\left(0, X^{*}\right)$, then

$$
\int_{\mathbb{R}} K(x-y) H(U(y)-\theta) d y=\int_{-\infty}^{x-X^{*}} K(\xi) d \xi+\int_{x}^{\infty} K(\xi) d \xi \equiv g_{2}(x) .
$$

(III) If $U<\theta$ on $(-\infty, 0)$ and $U>\theta$ on $(0,+\infty)$, then

$$
\int_{\mathbb{R}} K(x-y) H(U(y)-\theta) d y=\int_{-\infty}^{x} K(\xi) d \xi \equiv g_{3}(x) .
$$

(IV) If $U>\theta$ on $(-\infty, 0)$ and $U<\theta$ on $(0,+\infty)$, then

$$
\int_{\mathbb{R}} K(x-y) H(U(y)-\theta) d y=\int_{x}^{\infty} K(\xi) d \xi \equiv g_{4}(x) .
$$

Suppose that $\phi^{\prime}>0$ so that it is strictly increasing and smooth. Then $\phi^{\prime}(U)+$ $\gamma g_{i}>0$ for $U>0$ and $\gamma \geq 0$. Applying the implicit function theorem, the existence and uniqueness of a steady-state are proved. By using the equation $\phi(U)=(\alpha-\gamma U) g_{i}(x)$, if $U(\Omega)>\beta$ at some $\Omega \in \mathbb{R}$, recall that $\beta>0$ is the solution of the equation $\phi(U)=\alpha-\gamma U$, then we obtain a contradiction $g_{i}(\Omega)>1$ 
immediately. Therefore $U \leq \beta$ on $\mathbb{R}$. We have to verify the above prescribed conditions for each case.

Case 1: Let $\alpha-\gamma \theta>2 \phi(\theta)$. By using fundamental analysis, there exists a unique number $X_{*}>0$, such that $\phi(\theta)=(\alpha-\gamma \theta) \int_{0}^{X_{*}} K(x) d x$. By differentiating the equation

about $x$, we have

$$
\phi(U)=(\alpha-\gamma U) \int_{x-X_{*}}^{x} K(\xi) d \xi
$$

$$
\phi^{\prime}(U) U^{\prime}+\gamma \int_{x-X_{*}}^{x} K(\xi) d \xi U^{\prime}=(\alpha-\gamma U)\left[K(x)-K\left(x-X_{*}\right)\right] .
$$

Hence

$$
U^{\prime}=(\alpha-\gamma U)\left[K(x)-K\left(x-X_{*}\right)\right] /\left[\phi^{\prime}(U)+\gamma \int_{x-X_{*}}^{x} K(\xi) d \xi\right] .
$$

It is easy to show that $\alpha-\gamma U \geq \alpha-\beta \gamma>0$ and that if $K^{\prime}<0$ on $\mathbb{R}^{+}$, then $U^{\prime}>0$ for $x<\frac{X_{*}}{2}$ and $U^{\prime}<0$ for $x>\frac{X_{*}}{2}$. Clearly $U(0)=U\left(X_{*}\right)=\theta$. The proof is finished.

Case 2: Let $\alpha-\gamma \theta<2 \phi(\theta)$. As in Case 1, there is a unique number $X^{*}>0$, such that $\phi(\theta)=(\alpha-\gamma \theta)\left[\int_{-\infty}^{-X^{*}} K(x) d x+\int_{0}^{\infty} K(x) d x\right]$. As in Case 1 , by

$$
\phi(U)=(\alpha-\gamma U)\left[\int_{-\infty}^{x-X^{*}} K(\xi) d \xi+\int_{x}^{\infty} K(\xi) d \xi\right],
$$

we have

$$
U^{\prime}=(\alpha-\gamma U)\left[K\left(x-X^{*}\right)-K(x)\right] /\left\{\phi^{\prime}(U)+\gamma \int_{-\infty}^{x-X^{*}} K(\xi) d \xi+\gamma \int_{x}^{\infty} K(\xi) d \xi\right\} .
$$

So if $K^{\prime}<0$ on $\mathbb{R}^{+}$, then $U^{\prime}<0$ for $x<\frac{X^{*}}{2}$ and $U^{\prime}>0$ for $x>\frac{X^{*}}{2}$.

Case 3: Let $\alpha-\gamma \theta=2 \phi(\theta)$. Note that $\int_{-\infty}^{0} K(x) d x=\frac{1}{2}$. By using implicit differentiation on the equation

$$
\phi(U)=(\alpha-\gamma U) \int_{-\infty}^{x} K(\xi) d \xi
$$

we get

$$
\phi^{\prime}(U) U^{\prime}=(\alpha-\gamma U) K(x)-\gamma U^{\prime} \int_{-\infty}^{x} K(\xi) d \xi
$$

Thus

$$
U^{\prime}=(\alpha-\gamma U) K(x) /\left[\phi^{\prime}(U)+\gamma \int_{-\infty}^{x} K(\xi) d \xi\right] \geq 0 .
$$

Case 4: Let $\alpha-\gamma \theta=2 \phi(\theta)$. Similar to Case $3, \int_{0}^{\infty} K(x) d x=\frac{1}{2}$. From

$$
\phi(U)=(\alpha-\gamma U) \int_{x}^{\infty} K(\xi) d \xi
$$

we have

$$
U^{\prime}=-(\alpha-\gamma U) K(x) /\left[\phi^{\prime}(U)+\gamma \int_{x}^{\infty} K(\xi) d \xi\right] \leq 0
$$


Therefore, altogether we have found four steady-states under different conditions.

(B). Suppose that $\phi(U)=U(U-1)(U-a)$ and $0<a<1$. Then, the existence problem reduces to

$$
U^{3}-(1+a) U^{2}+\left[a+\gamma g_{i}(x)\right] U-\alpha g_{i}(x)=0 .
$$

Based on a fundamental formula for third-order polynomial equations, we can find the formal steady-state solutions for the four cases as follows

$$
U_{i}(x)=\frac{1+a}{3}-\{\xi(x)+\eta(x)\}^{1 / 3}-\{\xi(x)-\eta(x)\}^{1 / 3},
$$

where $i=1,2,3,4$; and

$$
\xi(x)=-\frac{\alpha}{2} g_{i}(x)+\frac{1}{6}(1+a)\left[a+\gamma g_{i}(x)\right]-\frac{1}{27}(1+a)^{3},
$$

and

$$
\begin{aligned}
\eta(x)= & \left\{\left[-\frac{\alpha}{2} g_{i}(x)+\frac{1}{6}(1+a)\left(a+\gamma g_{i}(x)\right)-\frac{1}{27}(1+a)^{3}\right]^{2}\right. \\
& \left.+\left[\frac{1}{3}\left(a+\gamma g_{i}(x)\right)-\frac{1}{9}(1+a)^{2}\right]^{3}\right\}^{1 / 2} .
\end{aligned}
$$

The analysis to select the unique number $X_{*}$ or $X^{*}$ is very similar to the case $\phi$ being strictly increasing.

Now, let the kernel be a Mexican hat function. We can prove the existence and uniqueness of $X_{*}$ and $X^{*}$, respectively, such that

$$
\begin{aligned}
& \phi(\theta)=(\alpha-\gamma \theta) \int_{0}^{X_{*}} K(x) d x, \text { if } \alpha-\gamma \theta>2 \phi(\theta) \text { and } X_{*}<+\infty, \\
& \phi(\theta)=(\alpha-\gamma \theta)\left[\int_{-\infty}^{-X^{*}} K(x) d x+\int_{0}^{\infty} K(x) d x\right], \text { if } \alpha-\gamma \theta<2 \phi(\theta) \text { and } X^{*}<+\infty, \\
& \phi(\theta)=(\alpha-\gamma \theta) \int_{-\infty}^{0} K(x) d x, \text { if } \alpha-\gamma \theta=2 \phi(\theta) \text { and } X_{*}=+\infty, \\
& \phi(\theta)=(\alpha-\gamma \theta) \int_{0}^{\infty} K(x) d x, \text { if } \alpha-\gamma \theta=2 \phi(\theta) \text { and } X^{*}=+\infty .
\end{aligned}
$$

Without loss of generality, let $\gamma=0$ and $\phi$ be an increasing function. The existence problem becomes $\phi(U)=\alpha g_{i}(x)$ and the steady-state is $U=\phi^{-1}\left(\alpha g_{i}(x)\right)$, for $i=1,2,3,4$.

Because of translation invariance of the steady-states, $\lambda=0$ is a neutral eigenvalue of the differential operator $\mathcal{L}$. However, unless $X_{*}=+\infty$ or $X^{*}=+\infty$, there is a positive eigenvalue, thus the steady-states are not stable. Therefore the proof is finished.

Acknowledgment: The author is very grateful to Professor David Terman (The Ohio State University) for his encouragement. 


\section{References}

1. Shun-ichi Amari, Dynamics of pattern formation in lateral-inhibition type neural fields. Biological Cybernetics, 27(1977), 77-87.

2. Paul C. Bressloff, S. E. Folias, Alain Prat and Yue-Xian Li, Oscillatory waves in inhomogeneous neural media. Physical Review Letters, 91(2003), 178101.

3. Xinfu Chen, Existence, uniqueness, and asymptotic stability of traveling waves in nonlocal evolution equations. Advances in Differential Equations, 2(1997), 125-160.

4. Stephen Coombes, Gabriel J. Lord and M R. Owen, Waves and bumps in neuronal networks with axo-dendritic synaptic interactions. Physica D, 178(2003), 219-241. Evans functions for integral neural field equations with Heaviside firing rate function. SIAM Journal on Applied Dynamical Systems. submitted.

5. G. Bard Ermentrout, Neural networks as spatio-temporal pattern-forming systems. Institute of Physics (Great Britain) Reports on Progress in Physics, 61(1998), 353-430.

6. G. Bard Ermentrout and J. Bryce McLeod, Existence and uniqueness of travelling waves for a neural network. Proceedings of the Royal Society of Edinburgh, 123A(1993), 461-478.

7. John W. Evans, Nerve axon equations: Indiana University Mathematics Journal, I Linear approximations. 21(1972), 877-885. II Stability at rest. 22(1972), 75-90. III Stability of the nerve impulse, 22(1972), 577-593. IV The stable and the unstable impulse. 24(1975), 11691190.

8. David J. Pinto and G. Bard Ermentrout, Spatially structured activity in synaptically coupled neuronal networks. I. traveling fronts and pulses, II. Lateral inhibition and standing pulses. SIAM Journal on Applied Mathematics, 62(2001), I. 206-225, II. 226-243.

9. Clifford Henry Taubes, Modeling Defferential Equations in Biology, Prentice Hall, Inc., 2001.

10. David Terman, Dynamics of singularly perturbed neuronal networks. An introduction to mathematical modeling in physiology, cell biology, and immunology (New Orleans, LA, 2001), 132, Proceedings of Symposium in Applied Mathematics, 59, American Mathematical Society, Providence, RI, 2002.

11. David Terman, An introduction to dynamical systems and neuronal dynamics. to appear in Proceedings of the MBI Program on Computational Neuroscience.

12. J. M. T. Thompson and H. B. Stewart, Nonlinear Dynamics and Chaos, Second Edition, John Wiley and Sons, 2002.

13. Linghai Zhang, On stability of traveling wave solutions in synaptically coupled neuronal networks. Differential and Integral Equations, 16(2003), 513-536.

14. Linghai Zhang, Existence, uniqueness and exponential stability of traveling wave solutions of some integral differential equations arising from neuronal networks. Journal of Differential Equations, 197(2004), 162-196.

15. Linghai Zhang, et al, Connecting orbits in nonlocal excitatory neuronal networks. Preprint.

Department of Mathematics, Lehigh University, Bethlehem, Pennsylvania USA 18015

E-mail address: liz5@lehigh.edu 